\title{
Die Kaskadenschaltung zweier mehrphasiger Induktionsmaschinen in analytischer und graphischer Behandlung.
}

\author{
Von \\ Dr. Ing. H. Kafka.
}

I. Ersatzstromkreis der mehrphasigen Induktionsmaschine. Für die mehrphasige Induktionsmaschine (allgemeiner Wechselstromtransformator) gilt bei Reduktion der sekundären Größen auf die primäre Windungszahl, Wicklungsart und Kreisfrequenz pro Phase der Ersatzstromkreis Abb. I. Dabei sind die Eisenverluste im Ständer und Läufer in einer den wirklichen Verhältnissen nahe kommenden Weise berücksichtigt.

Bezüglich der verwendeten Bezeichnungen ist $\mathrm{zu}$ bemerken, daß vektorielle Größen mit deutschen Buchstaben, ihre absoluten Werte mit lateinischen Buchstaben bezeichnet wurden. Die Operationszeichen in Gleichungen mit vektoriellen Größen sind vektoriell aufzufassen. Alle Entwicklungen gelten, wenn nicht anders bemerkt, pro Phase.

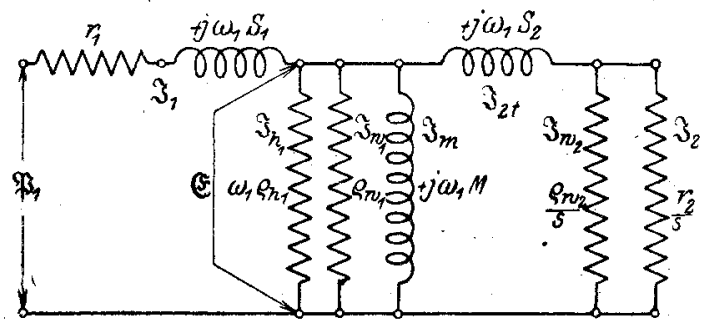

Abb. I.

Bezeichnungen:

$\mathfrak{P}_{1}$ Effektivwert der primär zugeführten Klemmenspannung.

$\mathbb{F}_{1}$ dem Ständerfluß $\Phi_{1}$ entsprechende Spannung.

(F) dem gemeinsamen Fluß $\Phi$ entsprechende Spannung.

$\mathfrak{F}_{2}$ dem Läuferfluß $\Phi_{2}$ entsprechende Spannung.

$r_{1}, S_{1}$ Wirkwiderstand und Streuinduktionskoeffizient der Ständerwicklung.

$r_{2}, S_{2}$ reduzierter Wirkwiderstand und Streuinduktionskoeffizient der Läuferwicklung.

M reduzierter gegenseitiger Induktionskoeffizient der beiden Wicklungen der Induktionsmaschine.

$\omega_{1}$ Kreisfrequenz der Ständerströme.

$\omega_{2}$ Kreisfrequenz der Läuferströme.

$\mathrm{s}=\frac{\omega_{1}}{\omega_{2}}$ Schlüpfung.

$\omega_{1} \varrho_{\mathrm{h}_{1}}$ fiktiver Wirkwiderstand, welcher die Hysteresisverluste im Ständereisen (prop. $\omega_{1} \Phi_{1}^{2}$ ) berücksichtigt.

$\varrho_{\mathrm{w}_{1}}$ fiktiver Wirkwiderstand, welcher die Wirbelströme im Ständereisen (prop. $\omega_{1}^{2} \Phi_{1}^{2}$ ) berücksichtigt.

$\pm \omega_{1} \varrho_{\mathrm{h} 2}$ fiktiver Wirkwiderstand, welcher die Hysteresisverluste im Läufereisen berücksichtigt; das doppelte Vorzeichen soll den Hysteresissprung zum Ausdruck bringen und zwar gilt das \pm Zeichen für $s \gtreqless 0$.

$\varrho_{\mathrm{w} 2}$ fiktiver Wirkwiderstand, welcher die Wirbelströme im Läufereisen berücksichtigt. 
Die Berücksichtigung der Läuferhysteresis hat zur Folge, daß die Leistungs linie im Kreisdiagramm der Induktionsmaschine keine Gerade ist. Zur Vereinfachung empfiehlt es sich, die Läuferhysteresis zu vernachlässigen und die den Ständereisenverlusten entsprechenden fiktiven Wirkwiderstände $\omega_{1} \varrho_{\mathrm{h}_{1}}$ und $\varrho_{\mathrm{w}_{1}}$ im Ersatzstromkreis parallel zum Blindwiderstand $\mathrm{j} \omega_{1} \mathrm{M}$ zu legen (siehe Abb. 2); die genannten Eisenverluste werden damit auf die dem gemeinsamen Fluß $\Phi$ entsprechenden Induktionen bezogen.

2. Drehzahl- und Schlüpfungsverhältnisse der Kaskadenschaltung. Die beiden in Kaskade geschalteten Induktionsmaschinen sollen durch die Bezeichnung ,vordere" und "hintere" Maschine unterschieden werden. Die Ständerwicklung der vorderen Maschine sei an das Mehrphasennetz angeschlossen; ihre Läuferwicklung kann entweder mit der Ständer- oder mit der Läuferwicklung der hinteren Maschine verbunden sein, wobei auf die richtige Verbindung der einzelnen Phasen zu achten ist.

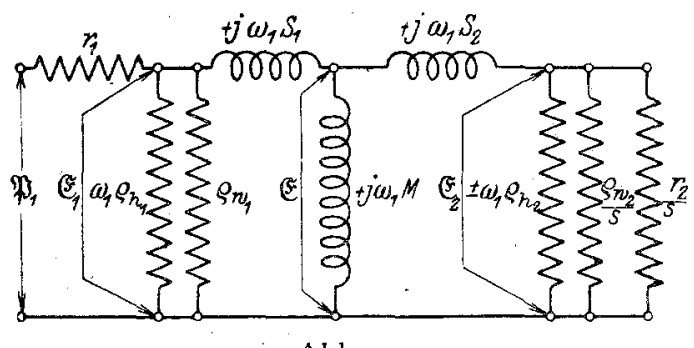

Abb. 2.

Die beiden Maschinen mit den Polpaarzahlen $\mathrm{p}_{1}$ und $\mathrm{p}_{2}$ seien mit dem Übersetzungsverhältnis

$$
\gamma=\frac{\mathrm{n}_{1}}{\mathrm{n}_{2}}
$$

gekuppelt; $\mathrm{n}_{1}$ und $\mathrm{n}_{2}$ sind die minutlichen Umdrehungszahlen ihrer Läufer.

Die der Ständerwicklung der vorderen Maschine zugeführten Mehrphasenströme der Periodenzahl $f_{1}$ erzeugen ein Drehfeld mit der minutlichen Umdrehungszahl

$$
\mathrm{N}_{1}=\frac{60 \mathrm{f}_{1}}{\mathrm{p}_{1}}
$$

der Differenz der Umdrehungszahlen $\left(\mathrm{N}_{1}-\mathrm{n}_{1}\right)$ entspricht die Periodenzahl

$$
\mathrm{f}_{2}=\frac{\left(\mathrm{N}_{1}-\mathrm{n}_{1}\right) \mathrm{p}_{1}}{60}
$$

der Mehrphasenströme, welche in den hintereinander geschalteten Wicklungen der beiden Maschinen fließen. In der hinteren Maschine erzeugen diese Ströme ein Drehfeld mit der minutlichen Umdrehungszahl

$$
\mathrm{N}_{2}=\frac{60 \mathrm{f}_{2}}{\mathrm{p}_{2}}=\left(\mathrm{N}_{1}-\mathrm{n}_{1}\right) \cdot \frac{\mathrm{p}_{1}}{\mathrm{p}_{2}}
$$

der Differenz der Umdrehungszahlen $\left(\mathrm{N}_{2}-\mathrm{n}_{2}\right)$ entspricht die Periodenzahl

$$
\mathrm{f}_{3}=\frac{\left(\mathrm{N}_{2}-\mathrm{n}_{2}\right) \mathrm{p}_{2}}{6 \mathrm{o}}
$$

der in der Sekundärwicklung der hinteren Maschine fließenden Mehrphasenströme.

Wir haben demnach bei der Kaskadenschaltung 3 Stromkreise zu unterscheiden, in denèn Mehrphasenströme 'dèr Periodenzahlen $f_{1}, f_{2}$ und $f_{3}$ fließen. Der „Primärkreis der Kaskadenschaltung" besteht aus der Ständerwicklung der vorderen Maschine (primäre Periodenzahl $f_{1}$ ), der "Sekundärkreis" aus den hintereinander geschalteten Wicklungen der beiden Maschinen (sekundäre Periodenzahl $\mathrm{f}_{2}$ ) und der ,Tertiärkreis" aus der Sekundärwicklung der hinteren Maschine (tertiäre Periodenzahl $f_{\mathbf{3}}$ ). 
Wenn der Läufer der hinteren Maschine synchron mit ihrem Drehfeld rotiert $\left(n_{2}=N_{2}\right)$, wird $f_{3}=0$; dieser Betriebszustand wird als Synchronismus der Kaskadenschaltung bezeichnet. Die Bedingungsgleichung für die synchronen Umdrehungszahlen der beiden Läufer $n_{10}$ und $n_{20}$ ist nach 4 )

da nach I)

$$
\mathrm{n}_{20}=\left(\mathrm{N}_{1}-\mathrm{n}_{10}\right) \cdot \frac{\mathrm{p}_{1}}{\mathrm{p}_{2}}
$$

wird

$$
\frac{\mathrm{n}_{10}}{\mathrm{n}_{20}}=\gamma
$$

und

$$
\mathrm{n}_{10}=\mathrm{N}_{1} \cdot \frac{\gamma \mathrm{p}_{1}}{\gamma \mathrm{p}_{1}+\mathrm{p}_{2}}=\gamma \cdot \frac{60 \mathrm{f}_{1}}{\gamma \mathrm{p}_{1}+\mathrm{p}_{2}}
$$

$$
\mathrm{n}_{20}=\mathrm{N}_{1} \cdot \frac{\mathrm{p}_{1}}{\gamma \mathrm{p}_{1}+\mathrm{p}_{2}}=\frac{60 \mathrm{f}_{1}}{\gamma \mathrm{p}_{1}+\mathrm{p}_{2}} \text {. }
$$

Nach Einführung der Verhältniszahlen

$$
\begin{aligned}
& \alpha=\frac{\gamma \mathrm{p}_{1}}{\gamma \mathrm{p}_{1}+\mathrm{p}_{2}}, \quad(\alpha+\beta=\mathrm{I}) \\
& \beta=\frac{\mathrm{p}_{2}}{\gamma \mathrm{p}_{1}+\mathrm{p}_{2}}(\alpha)
\end{aligned}
$$

lassen sich die synchronen Umdrehungszahlen in der Form

und

$$
\mathrm{n}_{10}=\alpha \mathrm{N}_{1}
$$

schreiben.

$$
\mathrm{n}_{20}=\frac{\alpha \mathrm{N}_{1}}{\gamma}=\beta \cdot \frac{\mathrm{p}_{1}}{\mathrm{p}_{2}} \cdot \mathrm{N}_{1}
$$

Als Schlüpfung der Kaskadenschaltung wird das Verhältnis

$$
\mathrm{s}=\frac{\mathrm{n}_{10}-\mathrm{n}_{1}}{\mathrm{n}_{10}}=\frac{\mathrm{n}_{20}-\mathrm{n}_{2}}{\mathrm{n}_{20}}
$$

der Differenz zwischen der synehronen und der tatsächlichen Umdrehungszahl einer Maschine zu ihrer synchronen Umdrehungszahl bezeichnet.

Die Eigenschlüpfungen der beiden Maschinen sind

und

$$
\mathrm{s}_{1}=\frac{\mathrm{N}_{1}-\mathrm{n}_{1}}{\mathrm{~N}_{1}}
$$

Da

$$
\mathrm{s}_{2}=\frac{\mathrm{N}_{2}-\mathrm{n}_{2}}{\mathrm{~N}_{2}}
$$

und

$$
\mathrm{n}_{1}=\mathrm{n}_{10}(\mathrm{I}-\mathrm{s})
$$

$$
\mathrm{N}_{1}=\frac{\mathrm{n}_{10}}{\alpha}(6 \mathrm{a})
$$

wird

$$
\frac{\mathrm{n}_{1}}{\mathrm{~N}_{1}}=\alpha(\mathrm{I}-\mathrm{s})
$$

und damit die Eigenschlüpfung der vorderen Maschine (da $I-\alpha=\beta$ )

$$
\mathrm{s}_{1}=\mathrm{I}-\frac{\mathrm{n}_{1}}{\mathrm{~N}_{1}}=\alpha \mathrm{s}+\beta \text {. }
$$

Um die Eigenschlüpfung der hinteren Maschine $s_{8}$ durch $s$ und die Konstanten $\alpha$ und $\beta$ auszudrücken, ermitteln wir zunächst aus 4) und IOa) das Verhältnis 


$$
\frac{\mathrm{N}_{2}}{\mathrm{~N}_{1}}=\left(\mathrm{I}-\frac{\mathrm{n}_{1}}{\mathrm{~N}_{1}}\right) \cdot \frac{\mathrm{p}_{1}}{\mathrm{p}_{2}}=(\alpha \mathrm{S}+\beta) \cdot \frac{\mathrm{p}_{1}}{\mathrm{p}_{2}}
$$

wenn wir in 7 a) $\mathrm{N}_{1}$ durch $\mathrm{N}_{2}$ ausdrücken, wird die synchrone Umdrehungszahl der hinteren Maschine

Aus den Beziehungen

$$
\mathrm{n}_{20}=\frac{\beta}{\alpha \mathrm{s}+\beta} \cdot \mathrm{N}_{2}
$$

$$
\mathrm{n}_{2}=\mathrm{n}_{20}(\mathrm{I}-\mathrm{s}), \mathrm{N}_{2}=\mathrm{n}_{20} \cdot \frac{\alpha \mathrm{s}+\beta}{\beta}(7 \mathrm{~b}) \text { und } \frac{\mathrm{n}_{2}}{\mathrm{~N}_{2}}=\frac{\beta(\mathrm{I}-\mathrm{s})}{\alpha \mathrm{s}+\beta}
$$

ergibt sich schließlich die Eigenschlüpfung der hinteren Maschine

$$
\mathrm{s}_{2}=\mathrm{I}-\frac{\mathrm{n}_{2}}{\mathrm{~N}_{2}}=\frac{\mathrm{s}}{\alpha \mathrm{s}+\beta} \text {. }
$$

Das Produkt der Eigenschlüpfungen ist

$$
\mathrm{s}_{1} \mathrm{~s}_{2}=\mathrm{s} \text {. }
$$

Für die Schlüpfung $\mathrm{s}=-\frac{\beta}{\alpha}$ wird die Eigenschlüpfung der vorderen Maschine $s_{1}=0$, d. h. ihr Läufer rotiert synchron mit dem Drehfeld $\left(n_{1}=N_{1}\right)$. Im folgenden wird dieser Betriebszustand als „Synchronismus der vorderen Maschine“ bezeichnet.

Die Eigenschlüpfung der vorderen Maschine bestimmt auch das Verhältnis der sekundären zur primären Periodenzahl (bzw. Kreisfrequenz)

$$
\mathrm{s}_{1}=\frac{\mathrm{f}_{2}}{\mathrm{f}_{1}}=\frac{\omega_{2}}{\omega_{1}}=\alpha \mathrm{s}+\beta
$$

die Eigenschlüpfung der hinteren Maschine bestimmt das Verhältnis der tertiären zur sekundären Periodenzahl

$$
\mathrm{s}_{2}=\frac{\mathrm{f}_{3}}{\mathrm{f}_{2}}=\frac{\omega_{3}}{\omega_{2}}=\frac{\mathrm{s}}{\alpha \mathrm{s}+\beta} .
$$

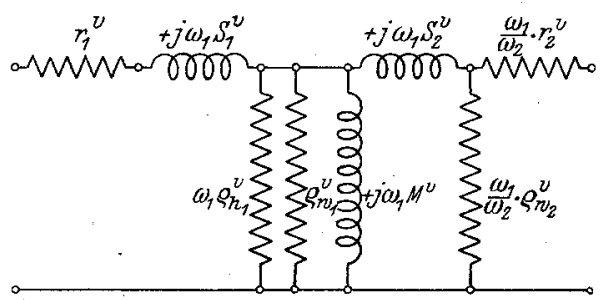

Abb. 3 a.

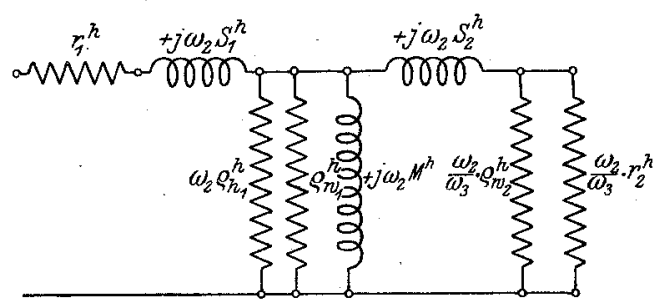

Abb. 3 b.

Die Multiplikation von 13) und I4) ergibt das Verhältnis der tertiären zur primären Periodenzahl (bzw. Kreisfrequenz)

$$
\frac{\mathrm{f}_{3}}{\mathrm{f}_{1}}=\frac{\omega_{3}}{\omega_{1}}=\mathrm{s}
$$

Bei direkter Kupplung der Läuferwellen $(\gamma=1)$ wird

und die synchrone Umdrehungszahl

$$
\mathrm{n}_{1}=\mathrm{n}_{2}=\mathrm{n}
$$

ferner ist dann

$$
\mathrm{n}_{0}=\frac{60 \mathrm{f}_{1}}{\mathrm{p}_{1}+\mathrm{p}_{2}}
$$

$$
\begin{aligned}
& \alpha=\frac{\mathrm{p}_{1}}{\mathrm{p}_{1}+\mathrm{p}_{2}}, \\
& \beta=\frac{\mathrm{p}_{2}}{\mathrm{p}_{1}+\mathrm{p}_{2}} .
\end{aligned}
$$

3. Der Ersatzstromkreis der Kaskadenschaltung zweier mehrphasiger Induktionsmaschinen läßt sich aus den in den $\mathrm{Abb}$. 3 a und $3 \mathrm{~b}$ dargestellten Ersatzstrom- 
kreisen der vorderen und hinteren Maschine zusammensetzen, welche in ihrem Aufbaŭ der Abb. 2 entsprechen. Dabei sind die Konstanten der Sekundärwicklung der vorderen Maschine und beider Wicklungen der hinteren Maschine auf die Windungszahl und Wicklungsart der Primärwicklung der vorderen Maschine reduziert gedacht. Außerdem wurden die Kreisfrequenzen des Sekundärkreises jeder Maschine durch Multiplikation der sekundären Scheinwiderstände mit dem Verhältnis $\frac{\omega_{1}}{\omega_{2}}$ bzw. $\frac{\omega_{2}}{\omega_{3}}$ auf die Kreisfrequenz $\omega_{1}$ bzw. $\omega_{2}$ ihres Primärkreises reduziert. Die auf die beiden Maschinen bezughabenden Konstanten sind durch die oberen Zeiger , $\mathrm{V}^{\text {" }}$ bzw. „h" unterschieden. Alle Entwicklungen gelten wieder pro Phase.

Wenn der Ersatzstromkreis der hinteren. Maschine durch Multiplikation allẹ seiner Scheinwiderstände mit dem Verhältnis $\frac{\omega_{1}}{\omega_{2}}$ auf die Kreisfrequenz $\omega_{1}$ reduziert wird, lassen sich die Abb. 3 a und 3 b zu dem Ersatzstromkreis Abb. 4 zusammenfassen. Dabei empfiehlt es sich, zunächst den die Wirbelströme im Läufereisen der vorderen Maschine berücksichtigenden Wirkwiderstand $\frac{\omega_{1}}{\omega_{2}} \cdot \varrho_{\mathrm{w}_{2}}^{\mathrm{v}}$ zu vernachlässigen, da er eine durch seine Bedeutung nicht gerechtfertigte Komplikation der Behandlungsweise zur Folge hätte.

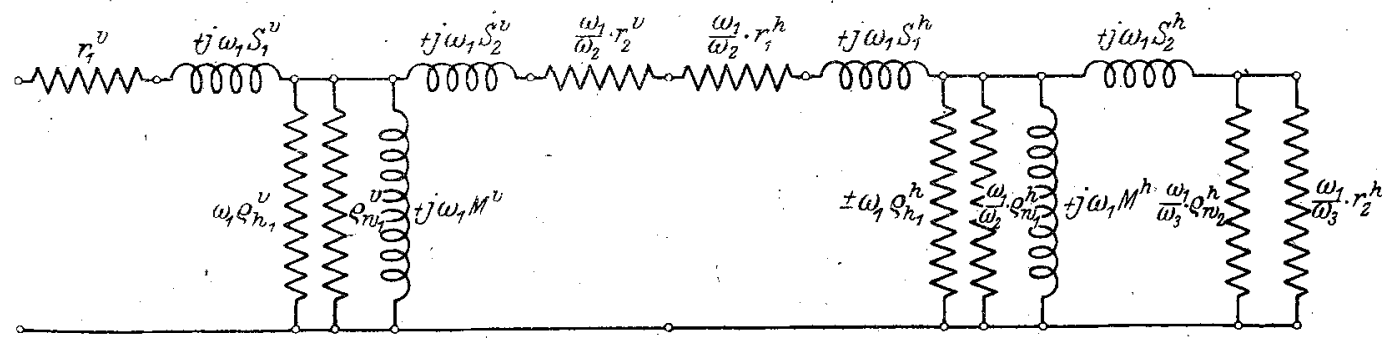

Abb. 4 .

Die Hysteresis und die Wirbelströme im Eisen des Primärteiles der hinteren Maschine liefern Beiträge zum Drehmoment. Beim Durchgang des Aggregates durch die Schlüpfung $\mathrm{s}=-\frac{\beta}{\alpha}$, bei welcher der Läufer der vorderen Maschine synchron mit ihrem Drehfeld rotiert $\left(\mathrm{n}_{1}=\mathrm{N}_{1}\right)$, kehrt sich die Richtung des Drehfeldes in der hinteren Maschine um. Im Zusammenhang damit ändern auch die genannten Beiträge ihren Sinn und zwar sind sie unterhalb der der Schlüpfung $s=-\frac{\beta}{\alpha}$ entsprechenden Umdrehungszahl positiv, oberhalb derșelben negativ. Die Änderung des Beitrages der Wirbelströme, die sich durch Vorzeichenänderung des Verhältnisses $\frac{\omega_{1}}{\omega_{2}}=\frac{I}{\alpha \mathrm{s}+\beta}$ ausdrückt; geht stetig vor sich; der Hysteresisbeitrag ändert dagegen ähnlich wie bei der einfachen Induktionsmaschine; sprungweise seinen Sinn. Um dies analytisch zum Ausdruck zu bringen, ist dem Wirkwiderstand $\omega_{1} \varrho_{\mathrm{h}_{1}}^{\mathrm{h}}$, welcher die Hysteresis im Primärteil der hinteren Maschine berücksichtigt, ein doppeltes Vorzeichen zu geben. Das „, "-Zeichen gilt für $\mathrm{n}_{1}<\mathrm{N}_{1}$, das "--.-Zeichen für $n_{1}>N_{1}$.

Die Berücksichtigung der Wirbelströme im Eisen des Primärteiles der hinteren Maschine hat im Ersatzstromkreis. Abb. 4 das Auftreten eines mit der Schlüpfung veränderlichen Wirkwiderstandes $\frac{\omega_{1}}{\omega_{2}} \cdot \varrho_{\mathrm{w}_{1}}^{\mathrm{h}}=\frac{\varrho_{\mathrm{w}_{1}}^{\mathrm{h}}}{\alpha \mathrm{s}+\beta}$ im Gefolge, welcher die Behand- 
lung der Kaskadenschaltung ziemlich erschwert. Die Hysteresis im Primärteil der hinteren Maschine bringt andererseits eine Unstetigkeit mit sich. Wir werden daher im folgenden die Hysteresis und die Wirbelströme im Eisen des Primärteiles der hinteren Maschine vernachlässigen, da dieselben in Wirklichkeit nur eine untergeordnete Rolle spielen.

Die drei parallel geschalteten Widerstände $\omega_{1} \varrho_{h 1}^{\mathrm{v}}, \varrho_{\mathrm{w} 1}^{\mathrm{v}}$ und $\mathrm{j} \omega_{1} \mathrm{M}^{\mathrm{v}}$ des Ersatzstromkreises Abb. 4 lassen, sich zu einem Scheinwiderstand $z_{a}=r_{a}+j x_{a}$ zusammenfassen; für den Scheinleitwert dieser Parallelschaltung gilt die Beziehung

$$
\mathrm{g}_{\mathrm{a}}-\mathrm{j} \mathrm{b}_{\mathrm{a}}=\left(\frac{\mathrm{I}}{\omega_{1} \varrho_{\mathrm{h}_{1}}^{\mathrm{v}}}+\frac{\mathrm{I}}{\varrho_{\mathrm{w}}^{\mathrm{v}}}\right)-\mathrm{j} \frac{\mathrm{I}}{\hat{\omega}_{1} \mathrm{M}^{\mathrm{v}}}
$$

die Komponenten des Scheinwiderstandes za sind daher

$$
\begin{aligned}
& \mathrm{r}_{\mathrm{a}}=\frac{\mathrm{g}_{\mathrm{a}}}{\mathrm{g}_{\mathrm{a}}^{2}+\mathrm{b}_{\mathrm{a}}^{2}}=\frac{\frac{\mathrm{I}}{\omega_{1} \varrho_{\mathrm{h}_{1}}^{v}}+\frac{\mathrm{I}}{\varrho_{\mathrm{w} 1}^{v}}}{\left(\frac{\mathrm{I}}{\omega_{1} \varrho_{\mathrm{h}_{1}}^{v}}+\frac{\mathrm{I}}{\varrho_{\mathrm{w} 1}^{\mathrm{v}}}\right)^{2}+\left(\frac{\mathrm{I}}{\omega_{1} \mathrm{M}^{v}}\right)^{2}} \\
& \mathrm{x}_{\mathrm{a}}=\frac{\mathrm{b}_{\mathrm{a}}}{\mathrm{g}_{\mathrm{a}}^{2}+\mathrm{b}_{\mathrm{a}}^{2}}=\frac{\frac{\mathrm{I}}{\omega_{1} \mathrm{M}^{v}}}{\left(\frac{\mathrm{I}}{\omega_{1} \varrho_{\mathrm{h}}^{v}}+\frac{\mathrm{I}}{\varrho_{\mathrm{w} 1}^{v}}\right)^{2}+\left(\frac{\mathrm{I}}{\omega_{1} \mathrm{M}^{v}}\right)^{2}}
\end{aligned}
$$

schließlich

Die Wirkwiderstände $\pm \omega_{1} \varrho_{\mathrm{h}^{1}}^{\mathrm{h}}$ und $\frac{\varrho_{\mathrm{w} 1 \mathrm{1}}^{\mathrm{h}}}{\alpha \mathrm{s}+\beta}$ werden vernachlässigt. Wir setzen

$$
\mathrm{j} \omega_{1} M^{2}=\mathrm{j} \mathrm{x}_{\mathrm{b}}
$$

welcher Blindwiderstand der einfachen Schreibweise wegen gelegentlich auch mit $\jmath_{\mathrm{b}}$ bezeichnet werden wird.

Wir führen weiter folgende abkürzende Bezeichnungen ein:

$$
\begin{aligned}
r_{1}^{\mathrm{v}}=\mathrm{r}_{1} ; \quad \omega_{1} \mathrm{~S}_{1}^{\mathrm{v}}=\mathrm{x}_{1}, \\
\mathrm{r}_{2}^{\mathrm{r}}+\mathrm{r}_{1}^{\mathrm{h}}=\mathrm{r}_{2} ; \quad \omega_{1}\left(\mathrm{~S}_{2}^{\mathrm{v}}+\mathrm{S}_{1}^{\mathrm{h}}\right)=\mathrm{x}_{2}, \\
\mathrm{r}_{2}^{\mathrm{h}}=\mathrm{r}_{3} ; \quad \omega_{1} \mathrm{~S}_{2}^{\mathrm{h}}=\mathrm{x}_{3} .
\end{aligned}
$$

Die Wirkwiderstände $r_{2}^{\mathrm{h}}=\mathrm{r}_{3}$ und $\varrho_{\mathrm{W}_{2}}^{\mathrm{h}}$ fasseǹ wir zu dem totalen sekundären Wirkwiderstand der hinteren Maschine

zusammen. Schließlich setzen wir noch

$$
r_{3 t}=\frac{I}{\frac{I}{r_{3}}+\frac{I}{\varrho_{w_{2}}^{h}}}
$$

$$
\begin{aligned}
\mathrm{\jmath}_{1} & =\mathrm{r}_{1}+\mathrm{jx}_{1}, \\
\mathrm{\jmath}_{2 \mathrm{~s}} & =\frac{\omega_{1}}{\omega_{2}} \cdot \mathrm{r}_{2}+\mathrm{jx_{2 }}=\frac{\mathrm{r}_{2}}{\alpha \mathrm{s}+\beta}+\mathrm{j} \mathrm{x}_{2}, \\
\mathrm{z}_{3} \mathrm{~s} & =\frac{\omega_{1}}{\omega_{3}} \cdot \mathrm{r}_{3 \mathrm{t}}+\mathrm{j} \mathrm{x}_{3}=\frac{\mathrm{r}_{3 \mathrm{t}}}{\mathrm{s}}+\mathrm{jx_{3 }} .
\end{aligned}
$$

Mit diesen Einführungen läßt sich der Ersatzstromkreis Abb. 4 in der vereinfachten Form Abb. 5 darstellen, welche der Ermittlung des Leitwert-(Strom-)diagramms der Kaskadenschaltung zugrunde gelegt werden soll. Dabei sind von den Eisenverlusten

I. die Wirbelströme und die Hysteresis im Ständer der vorderen Maschine, und

2. die Wirbelströme im Sekundärteil der hinteren Maschine in einer den wirklichen Verhältnissen nahe kommenden Weise berücksichtigt. Vernachlässigt sind dagegen: 
I. Wirbelströme und Hysteresis im Läufer der vorderen Maschine,

2. Wirbelströme und Hysteresis im Primärteil der hinteren Maschine und

3. Hysteresis im Sekundärteil der hinteren Maschine.

In dem von Prof. A r nold verwendeten Ersatzstromkreis der Kaskadenschaltung (siehe „Wechselstromtechnik" V. Band, I. Teil, S. 492), welcher im wesentlichen der Abb. 5 entspricht, werden die Stromzweige der Magnetisierungsströme durch die

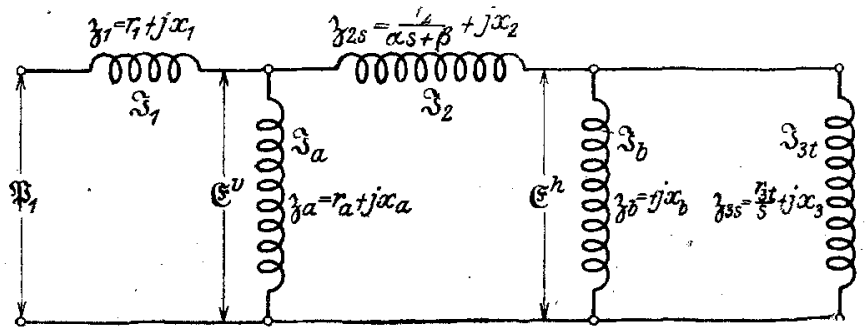

Abb. 5.

Scheinleitwerte $\mathfrak{Y}_{\mathrm{a}}=\mathrm{g}_{\mathrm{a}}+\mathrm{j} \mathrm{b}_{\mathrm{a}}$ und $\mathfrak{Y}_{\mathrm{b}}=\mathrm{g}_{\mathrm{b}}+\mathrm{j} \mathrm{b}_{\mathrm{b}}$ bestimmt. Wie aus dem Vergleich mit der Abb. 4 hervorgeht, kann durch die Wirkkomponente des letzteren nur die Hysteresis im Primärteil der hinteren Maschine berücksichtigt werden, wobei aber das Vorzeichen von $g_{b}$ zu wechseln ist, wenn die vordere Maschine relativ zu ihrem Drehfeld übersynchron läuft (S. 5).

\section{Ermittlung des Leitwertdiagramms der Kaskadenschaltung.}

Durch Zusammenfassung der Scheinwiderstände $z_{2 s}$, $\jmath_{\mathrm{b}}$ und $\tilde{\jmath}_{3 \mathrm{~s}}$ zu dem Scheinwiderstand

$$
\oslash_{2 s}=R_{2 s}+j X_{2 s}=z_{2 s}+\frac{z_{b} z_{3 s}}{z_{b}+z_{3 s}}
$$

kann der Ersatzstromkreis der Kaskadenschaltung auf die in Abb. 5 a dargestellte Form gebracht werden. Der resultierende Scheinleitwert desselben

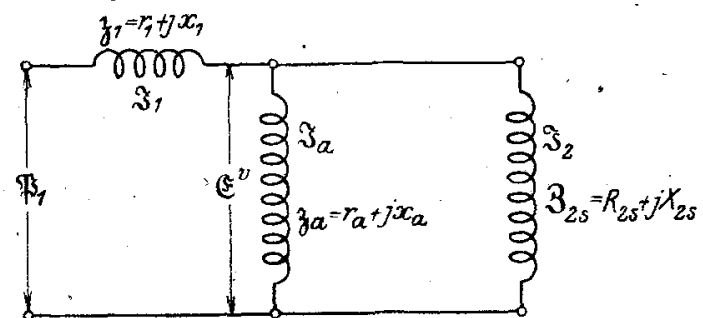

Abb. 5 a.

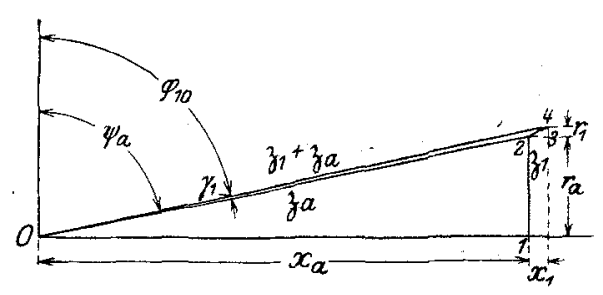

Abb. 6.

$$
\mathfrak{Y}_{\mathrm{s}}=\frac{z_{\mathrm{a}}+\mathfrak{Z}_{2 \mathrm{~s}}}{z_{1} z_{\mathrm{a}}+\mathfrak{z}_{1} \mathrm{~B}_{2 \mathrm{~s}}+z_{\mathrm{a}} \mathrm{Z}_{2 \mathrm{~s}}}
$$

läßt sich in einen konstanten Teil

$$
\mathfrak{Y}\left(-\frac{\beta}{\alpha}\right)=\frac{1}{z_{1}+z_{\mathrm{a}}}
$$

und einen mit der Schlüpfung s veränderlichen Teil

$$
\mathfrak{Y}_{\mathrm{s}}=\frac{1}{\left(\frac{z_{1}+z_{\mathrm{a}}}{z_{\mathrm{a}}}\right)^{2}\left(\frac{z_{1} z_{\mathrm{a}}}{z_{1}+z_{\mathrm{a}}}+z_{2 \mathrm{~s}}\right)}
$$

zerlegen. Mit Einführung des vektoriellen Verhältnisses

$$
\mathbb{E}_{1}=\frac{\hat{\partial}_{1}+\hat{z}_{\mathrm{a}}}{z_{\mathrm{a}}}
$$


und des Scheinwiderstandes

wird

$$
z_{1}^{*}=\frac{z_{1} z_{a}}{z_{1}+z_{a}}=\frac{z_{1}}{\tilde{E}_{1}}
$$

bzw.

$$
\mathfrak{Y}_{\mathrm{s}}=\frac{\mathrm{I}}{\mathbb{C}_{1}^{2}} \cdot \frac{\mathrm{I}}{3_{1}^{*}+3_{2} \mathrm{~s}}
$$

$$
\mathfrak{Y}_{\mathrm{s}}=\mathfrak{Y}_{\left(-\frac{\beta}{\alpha}\right)}+\mathfrak{Y}_{\mathrm{s}}=\frac{\mathrm{I}}{\mathfrak{z}_{1}+\mathfrak{z}_{\mathrm{a}}}+\frac{\mathrm{I}}{\widetilde{C}_{1}^{2}} \cdot \frac{\mathrm{I}}{\mathfrak{z}_{1}^{*}+\mathfrak{Z}_{2} \mathrm{~s}} .
$$

Wir wollen nun die Bedeutung der einzelnen Größen dieser Gleichung näher untersuchen. Für $\mathrm{s}=-\frac{\beta}{\alpha}$ (Synchronismus der vorderen Maschine) wird $z_{2 s}=\frac{r_{2}}{\alpha s+\beta}+\mathrm{jx}_{2}$ und damit $Z_{2 s}$ unendlich groß und der Ersatzstromkreis Abb. $5 \mathrm{a}$ reduziert sich auf die hintereinander geschalteten Scheinwiderstände $z_{1}$ und $z_{a}$; das erste Glied von 24a) stellt demnach den Scheinleitwert des Ersatzstromkreises für diese Schlüpfung dar.

Die Scheinwiderstände, welche das im zweiten Glied von 24a) auftretende vektorielle Verhältnis $\mathbb{E}_{1}$ bestimmen, sind in Abb. 6 spiegelbildlich ${ }^{1}$ ) eingetragen; es ist dabei das Verhältnis der Vektoren

und

$$
\frac{\overline{\mathrm{O}_{4}}}{\overline{\mathrm{O}_{2}}}=\frac{z_{1}+z_{\mathrm{a}}}{z_{\mathrm{a}}}
$$

$$
\Varangle\left(4 \mathrm{O}_{2}\right)=\gamma_{1} \text {. }
$$

Das in 27) eingeführte vektorielle Verhältnis kann auch in der Form geschrieben werden, wobei

$$
\mathfrak{S}_{1}=\mathrm{C}_{1} \mathrm{e}^{-\mathrm{j} \gamma_{1}}
$$

$$
C_{1}^{2}=\frac{\left(r_{1}+r_{a}\right)^{2}+\left(x_{1}+x_{a}\right)^{2}}{z_{a}^{2}}
$$

das Quadrat seines Betrages und $\gamma_{1}$ den Winkel bedeutet, um den ein Vektor bei der Multiplikation mit $\mathfrak{C}_{1}$ verdreht wird.

Nach Abb. 6 ist

und daher

$$
\gamma_{1}=\psi_{\mathrm{a}}-\varphi_{1 \mathrm{o}}
$$

$$
\operatorname{tg} \gamma_{1}=\frac{\frac{x_{a}}{r_{a}}-\frac{x_{1}+x_{a}}{r_{1}+r_{a}}}{1+\frac{x_{a}}{r_{a}} \cdot \frac{x_{1}+x_{a}}{r_{1}+r_{a}}} .
$$

Die Komponenten des in 28) eingeführten Scheinwiderstandes $z_{1}^{*}$ sollen mit $r_{1}^{*}$ und $x_{1}^{*}$ bezeichnet werden. Wenn in dieser Gleichung für $z_{1}$ und $z_{a}$ die komplexen Ausdrücke eingeführt werden, ergibt sich

$$
\begin{aligned}
z_{1}^{*}=r_{1}^{*}+j x_{1}^{*} & =\frac{\left(r_{1}+j x_{1}\right)\left(r_{a}+j x_{a}\right)}{\left(r_{1}+r_{a}\right)+j\left(x_{1}+x_{a}\right)} \\
& =\frac{r_{1} z_{a}^{2}+z_{1}^{2} r_{a}+j\left(x_{1} z_{a}^{2}+z_{1}^{2} x_{2}\right)}{\left(r_{1}+r_{a}\right)^{2}+\left(x_{1}+x_{a}\right)^{2}}
\end{aligned}
$$

1) Bezüglich der geometrischen Darstellung der Scheinleitwerte und Ströme ist zu bemerken, dafs dieselbe auf rechtwinkelige Koordinatensysteme bezogen wurde, wobei die pos. Wirkkomponenten in Richtung der pos. Ordinatenachse und die pos. (induktiven) Blindkomponenten in Richtung der neg. Abszissenachse des in der analytischen Geometrie gebräuchlichen Koordinatensystems aufgetragen wurden. Dementsprechend sind pos. (induktive) Blind widerstände in Richtung der neg. Abszissenachse aufzutragen. Zur Vereinfachung der auszuführenden Inversionen wurden jedoch die Scheinwiderstände an den Ordinatenachsen gespiegelt. 
und daraus

$$
\begin{aligned}
r_{1}^{*} & =\frac{r_{1} z_{a}^{2}+z_{1}^{2} r_{a}}{\left(r_{1}+r_{a}\right)^{2}+\left(x_{1}+x_{a}\right)^{2}}, \\
x_{1}^{*} & =\frac{x_{1} z_{a}^{2}+z_{1}^{2} x_{a}}{\left(r_{1}+r_{a}\right)^{2}+\left(x_{1}+x_{a}\right)^{2}} .
\end{aligned}
$$

$\mathrm{Da}$ nach $27 \mathrm{~b}$ ) für den Nenner dieser Ausdrücke die Beziehung

$$
\left(\mathrm{r}_{1}+\mathrm{r}_{\mathrm{a}}\right)^{2}+\left(\mathrm{x}_{1}+\mathrm{x}_{\mathrm{a}}\right)^{2}=\mathrm{C}_{1}^{2} z_{\mathrm{a}}^{2}
$$

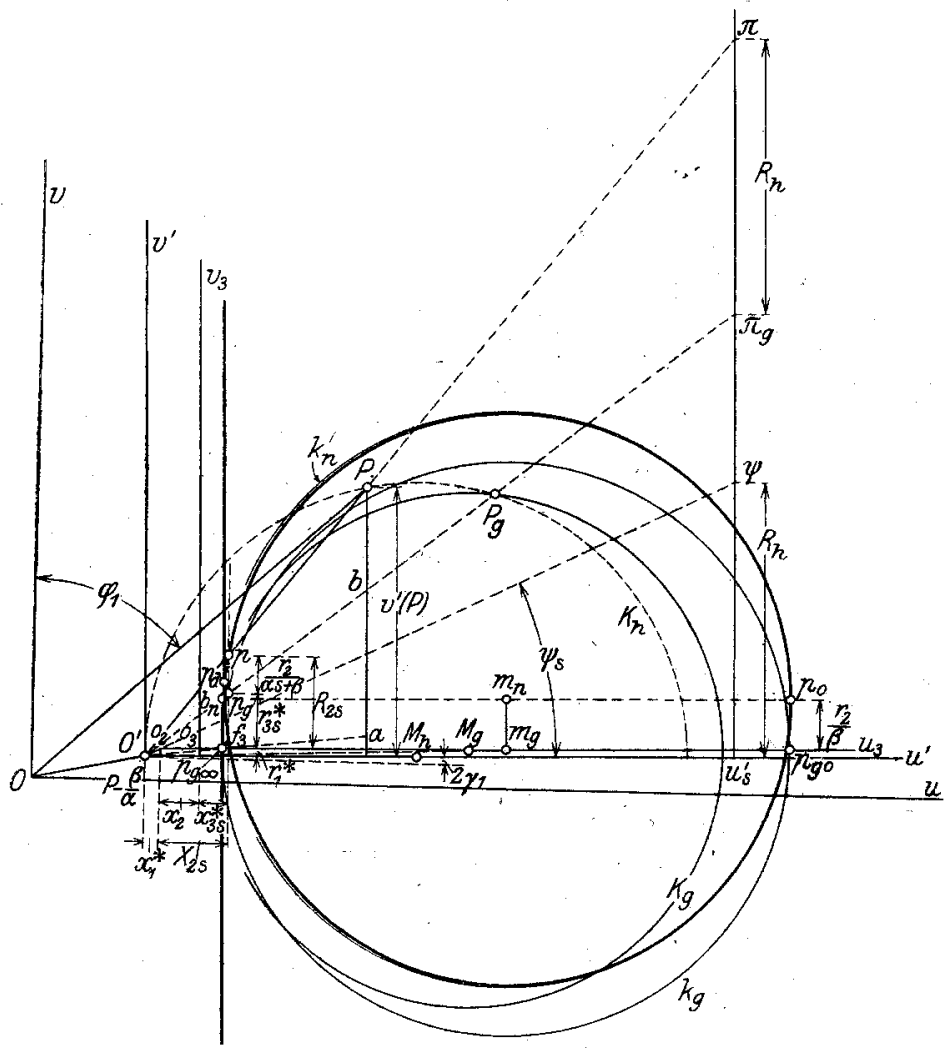

Abb. 7.

gilt, können die Komponenten von $z_{1}^{*}$ auch in der Form

geschrieben werden.

$$
\begin{aligned}
& r_{1}^{*}=\frac{1}{C_{1}^{2}} \cdot\left[r_{1}+r_{a}\left(\frac{z_{1}}{z_{a}}\right)^{2}\right], \\
& x_{1}^{*}=\frac{I}{C_{1}^{2}} \cdot\left[x_{1}+x_{a}\left(\frac{z_{1}}{z_{a}}\right)^{2}\right]
\end{aligned}
$$

Wir wollen nun den geometrischen Ort ermitteln, welchen der Vektor des Scheinleitwertes $\mathfrak{V}_{\mathrm{s}}=\mathfrak{V}_{\left(-\frac{\beta}{a}\right)}+\mathfrak{Y}_{\mathrm{s}}^{\prime}$ ( (siehe 24a) bei veränderlicher Schlüpfung s beschreibt. $\mathrm{Zu}$ diesem. Behufe tragen wir in dem Koordinatensystem (u v) der Abb. 7 zunächst den dem Synchronismus der vorderen Maschine entsprechenden konstanten Scheinleitwert

$$
\overline{\mathrm{OP}}_{-\frac{\beta}{\alpha}}=\frac{\mathbf{I}}{\bar{z}_{1}+z_{\mathrm{a}}}=\frac{\mathbf{I}}{\left(\mathrm{r}_{1}+\mathrm{r}_{\mathrm{a}}\right)+\mathrm{j}\left(\mathrm{x}_{1}+\mathrm{x}_{\mathrm{a}}\right)}
$$

ein. Der geometrische Ort des dem zweiten Gliede von 24 a) entsprechenden Scheinleitwertes 


$$
\mathfrak{Y}_{\mathrm{s}}=\frac{\mathrm{I}}{\mathrm{C}_{1}^{2}} \cdot \frac{\mathrm{I}}{\left(\mathfrak{Z}_{1}^{*}+8_{2 \mathrm{~s}}\right) \mathrm{e}^{-\mathrm{j} 2 \gamma_{1}}}
$$

kann durch Inversion aus den Scheinwiderständen $\left(\gamma_{1}^{*}+\delta_{2}\right) e^{-j 2 \gamma_{1}}$ abgeleitet werden, wobei die erhaltenen Scheinleitwerte noch im Verhältnis $\frac{\mathrm{I}}{\mathrm{C}_{1}^{2}}$ zu reduzieren sind. Wir führen hiezu ein neues Koordináténsystem ( $\left.u^{\prime} v^{\prime}\right)$ mit dem Ursprung $\mathrm{O}^{\prime} \equiv \mathrm{P}_{-\frac{\beta}{\alpha}}$ ein, welches gegen (uv) um den $\Varangle 2 \gamma_{1}$ entgegen dem Uhrzeigersinn verdreht ist und tragen in dasselbe die gespiegelten Scheinwiderstände $z_{1}^{*}+Z_{2 \mathrm{~s}}$ ein; auf (uv) bezogen, stellen diese Vektoren die gespiegelten Scheinwiderstände $\left(z_{1}^{*}+\beta_{2 s}\right) e^{-j 2 \gamma_{1}}$ dar

Die Scheinwiderstände $\left(z_{1}^{*}+B_{2}\right)$ setzen sich aus dem konstanten Teile

$$
z_{1}^{*}=r_{1}^{*}+j x_{1}^{*}
$$

und den mit der Schlüpfung $s$ veränderlichen Teilen

$$
乃_{2 \mathrm{~s}}=\mathrm{R}_{2 \mathrm{~s}}+\mathrm{jX} \text { is }
$$

zusammen, diese wieder nach 23) aus den Scheinwiderständen

und

$$
z_{2 \mathrm{~s}}=\frac{\mathrm{r}_{2}}{\alpha \mathrm{s}+\beta}+\mathrm{jx}_{2}
$$

$$
z_{3 s}^{*}=r_{3 s}^{*}+j x_{3 s}^{*}=\frac{z_{b} z_{3 s}}{z_{b}+z_{3 s}} .
$$

Wenn wir die konstanten von den mit der Schlüpfung veränderlichen. Teilen trennen, erhalten wir

$$
z_{1}^{*}+Z_{2 s}=r_{1}^{*}+j\left(x_{1}^{*}+x_{2}\right)+\frac{r_{2}}{\alpha s+\beta}+r_{3 s}^{*}+j x_{3 s}^{*} .
$$

In Abb. 7 ist dies dargestellt und zwar ist zunächst

und

$$
\overline{\mathrm{O}^{\prime} \mathrm{o}_{2}}=z_{1}^{*}=\mathrm{r}_{1}^{*}+\mathrm{j} \mathrm{x}_{1}^{*}
$$

$$
\mathrm{O}_{2} \mathrm{O}_{3}=\mathrm{x}_{2} \text {. }
$$

Der geometrische Ort des Scheinwiderstandes $z_{3 s}^{*}=r_{3 s}^{*}+j x_{3 s}^{*}$ der sich am einfachsten aus der Zerlegung

$$
\jmath_{3 s}^{*}=\jmath_{b}-\frac{z_{b}^{2}}{\jmath_{b}+z_{3 s}}=j x_{b}+\frac{x_{b}^{2}}{\frac{r_{3 t}}{s}+j\left(x_{b}+x_{3}\right)}
$$

ergibt, ist in dem Koordinatensystem $\left(u_{3} v_{3}\right) \|\left(u^{\prime} v^{\prime}\right)$ der Abb. 7 spiegelbildlich zur Darstellung gebracht. Für $s=0$ wird

$$
z_{30}^{*}=\mathrm{jx}_{\mathrm{b}}=\overline{\mathrm{o}_{3} \mathrm{pgo}_{\mathrm{o}}} \text {. }
$$

$\mathrm{Zu} \overline{\mathrm{O}_{3} \mathrm{Pgo}_{\mathrm{o}}}$ ist der Scheinwiderstand

$$
\overline{p_{g} \circ p_{g}}=\frac{x_{b}^{2}}{\frac{r_{3 t}}{s}+j\left(x_{b}+x_{3}\right)}
$$

zu addieren, der bei veränderlicher Schlüpfung einen Kreis $\mathrm{k}_{\mathrm{g}}$ mit dem Durchmesser

$$
2 r_{\mathrm{g}}=\frac{\mathrm{x}_{\mathrm{b}}^{2}}{\mathrm{x}_{\mathrm{b}}+\mathrm{x}_{3}} \text { (aus } 37 \text { ) zu ersehen) }
$$

beschreibt; der Mittelpunkt $\mathrm{m}_{\mathrm{g}}$ dieses Kreises liegt auf der $\mathrm{u}_{3}$-Achșe und hat vom Ursprung $\mathrm{O}_{3}$ den Abstand

$$
\overline{o_{3} m_{g}}=\overline{o_{3} p_{g o}}-r_{g}=x_{b}-\frac{1}{2} \cdot \frac{x_{b}^{2}}{x_{b}+x_{3}} .
$$


Aus 37) gehṭ hervor, daß für einen beliebigen Punkt $\mathrm{p}_{\mathrm{g}}$ des Kreises die Beziehung

$$
\operatorname{tg} \Varangle\left(\mathrm{pg}_{\mathrm{g} 0} \mathrm{p}_{3}\right)=\frac{\frac{\mathrm{r}_{3 \mathrm{t}}}{\mathrm{s}}}{\mathrm{x}_{\mathrm{b}}+\mathrm{x}_{3}}
$$

gilt, welche die Ermittlung der den verschiedenen Schlüpfungswerten entsprechenden Kreispunkte ermöglicht. Für $\mathrm{s}=\mathrm{I}$ (Stillstand) ist

$$
\operatorname{tg} \Varangle\left(\mathrm{p}_{\mathrm{g} \text { St }} \mathrm{p}_{\mathrm{g} 0} \mathrm{o}_{3}\right)=\frac{\mathrm{r}_{3 \mathbf{t}}}{\mathrm{x}_{\mathrm{b}}+\mathrm{x}_{\mathbf{3}}} ;
$$

der der Schlüpfung $s= \pm \infty$ entsprechende Punkt $p_{g_{\infty}}$ fällt wie $p_{g_{0}}$ in die $u_{s}$-Achse.

Die auf $\left(u_{3} v_{3}\right)$ bezogenen Ordinaten der Punkte $p_{g}$ stellen die Wirkwiderstände $r_{3 s}^{*}$, ihre Absżissen die Blindwiderstände $x_{3 s}^{*}$ dar. Um den geometrischen Ort der Scheinwiderstände $z_{x}^{*}+\oint_{\text {as }}$ zu erhalten, sind nach 34 ) noch die mit der Schlüpfung veränderlichen Wirkwiderstände

$$
\overline{\mathrm{p}_{\mathrm{g}} \mathrm{p}}=\frac{\mathrm{r}_{2}}{\alpha \mathrm{s}+\beta}
$$

von den Kreispunkten $p_{\mathbf{g}}$ parallel zur $v^{\prime}$-Achse aufzutragen. Die Verbindungslinie der so erhaltenen Punkte $p$ stellt, auf ( $u^{\prime} v^{\prime} i$ ) bezogen, das Spiegelbild des geometrischen Ortes der Scheinwiderstände $z^{*}+\mathcal{Z}_{2 s}$ dar (im folgenden ( $z_{x}^{*}+Z_{\text {as }}$ )-Kurve genannt).

Vom Punkte $\mathrm{p}_{\mathrm{g} 0}(\mathrm{~s}=0)$ ist der Wirkwiderstand $\overline{\mathrm{p}_{\mathrm{g} 0} \mathrm{p}_{0}}=\frac{\mathrm{r}_{2}}{\beta}$ und vom Punkte $\mathrm{p}_{\mathrm{gSt}}(\mathrm{s}=\mathrm{I})$ der Wirkwiderstand $\widetilde{\mathrm{pgst}_{\mathrm{gst}}}=\mathrm{r}_{2}$ aufzutragen. Für $\mathrm{s}= \pm \infty$ wird $\overline{p_{g \infty} p_{\infty}}=o, d$. h. der Punkt $p_{\infty} \operatorname{der}\left(z_{i}^{*}+\delta_{2 s}\right)$-Kurve fällt mit dem Punkte pgo des Kreises $k_{\mathbf{g}}$ zusammen.

Für $\mathrm{s}=-\frac{\beta}{\alpha}$ (Synchronismus der vorderen Maschine) wird $\alpha \mathrm{s}+\beta=0$ und der Wirkwiderstand $\frac{r_{2}}{\alpha s+\beta}$ unendlich groß; die in Abb. 7 durch den dieser Schlüpfung entsprechenden Kreispunkt $\mathrm{p}_{\mathrm{g}}\left(-\frac{\beta}{\alpha}\right)$ gezogene Parallele zur v'-Achse ist eine Asymptote $\operatorname{der}\left(z_{I}^{*}+B_{2 s}\right)$-Kurve.

Die $\left(z^{*}+Z_{2 s}\right)$-Kurve besitzt weiters einen Doppelpunkt $p_{d}$, welcher zwei Schlüpfungswerten $\pm s_{d}$ entspricht; für die ihn bestimmenden Scheinwiderstände gilt die Bedingung

$$
z_{2}\left(+s_{d}\right)+z_{3}^{*}\left(+s_{d}\right)=z_{2}\left(-s_{d}\right)+z_{3}^{*}\left(-s_{d}\right)
$$

Nach Einführung der komplexen Ausdrücke für die Scheinwiderstände $z_{a s}$ und $z_{3 s}^{*}$, wobei letzterer nach 35 ) zerlegt wird, ergibt sich

$$
\frac{r_{2}}{\alpha s+\beta}+j x_{2}+j x_{b}+\frac{x_{b}^{2}}{\frac{r_{3 t}}{s_{d}}+j\left(x_{b}+x_{3}\right)}=\frac{r_{2}}{-\alpha s_{d}+\beta}+j x_{2}+j x_{b}+\frac{x_{b}^{2}}{\frac{r_{3 t}}{-s_{d}}+j\left(x_{b}+x_{3}\right)}
$$

und daraus

$$
\mathrm{s}_{\mathrm{d}}= \pm \sqrt{\frac{\frac{\beta^{2}}{\alpha} \cdot \mathrm{x}_{\mathrm{b}}^{2}-\mathrm{r}_{2} \mathrm{r}_{3 \mathrm{t}}}{\alpha \mathrm{x}_{\mathrm{b}}^{2}+\frac{\mathrm{r}_{2}}{\mathrm{r}_{3 \mathrm{t}}} \cdot\left(\mathrm{x}_{\mathrm{b}}+\mathrm{x}_{\mathrm{3}}\right)^{2}}}
$$

Die Punkte des Kreises $k_{\mathrm{g}}$, welche kleinen Schlüpfungswerten entsprechen, erfüllen verhältnismäßig große Teile des Kreisumfanges zu beiden Seiten des Punktes $\mathrm{p}_{\mathrm{g} 0}$. Dies hat zur Folge, daß sich die Werte der von den Punkten $\mathrm{p}_{\mathrm{g}}$ aufzutragenden 
Wirkwiderstände $\frac{r_{2}}{\alpha s+\beta}$ auf diesen Teilen des Kreises nur wenig von dem Werte $\frac{r_{2}}{\beta}$ für $s=0$ unterscheiden; die $\left(z_{1}^{*}+\beta_{2 s}\right)$-Kurve wird sich daher in der Umgebung ihres Synchronismuspunktes $p_{0}$ dem Kreise $k_{n}$ mit dem Mittelpunkte $m_{n}$ nähern, welcher durch Verschiebung des Kreises $k_{\mathrm{g}}$ parallel zur v'-Achse um die Strecke $\frac{r_{2}}{\beta}$ erhalten werden kann. Wie aus Abb. 7 zu ersehen ist, schließt dieser Kreis die sich beiderseits des Synchronismuspunktes $p_{0}$ bis zum Doppelpunkte $p_{d}$ erstreckenden Teile der $\left(\partial^{*}+\zeta_{2 s}\right)$-Kurve ein.

Durch Inversion der $\left(3_{\mathrm{r}}^{*}+\mathrm{B}_{2 \mathrm{~s}}\right)$-Kurve in bezug auf den Pol O', wobei die erhaltenen Leitwerte nach 32) im Verhältnis $\frac{I}{C_{1}^{2}} \mathrm{zu}$ reduzieren sind, ergibt sich der geometrische Ort der Scheinleitwerte $\mathfrak{Y}$ 's bei veränderlicher Schlüpfung, welcher auf (u v) bezogen das gesuchte Leitwertdiagramm der Kaskadenschaltung darstellt. In Abb. 7.ist nur ein Punkt $P$ dieses Diagramms eingetragen, welcher aus dem entsprechenden Punkte der $\left(z_{I}^{*}+\beta_{2 s}\right)$-Kurve durch Inversion nach 32) abgeleitet zu denken ist, so daß

$$
\overline{\mathrm{O}^{\prime} \mathrm{P}}=\frac{\mathrm{I}}{\mathrm{C}_{1}^{3}} \cdot \frac{\mathrm{I}}{\overline{\mathrm{O}^{\prime} \mathrm{p}}}
$$

Das vorstehend beschriebene Verfahren für die Ermittlung des Kaskadendiagramms kann dadurch vereinfacht werden, daß direkt die Konstruktion der $\left(\partial_{\mathbf{r}}^{*}+\bigcap_{2 s}\right)$-Kurve in bezug auf den Punkt $O^{\prime}$ als Pol inversiert wird; bei der Inversion ist das in 32) auftretende Reduktionsverhältnis $\frac{\mathrm{I}}{\mathrm{C}_{1}^{2}}$ zu berücksichtigen.

Die Punkte der $\left(\gamma^{*}+\beta_{s s}\right)$-Kurve wurden durch Auftragen der Strecken $\overline{p_{g} p}=\frac{r_{2}}{\alpha s+\beta}$ von den Punkten $p_{g}$ des Kreises $k_{g}$ auf Parallelen zur $v$-Achse erhalten. Wir denken uns nun in Abb. 7 zunächst den genannten Kreis in bezug auf den Pol O' mit Berücksichtigung des Reduktionsverhältnisses $\frac{\mathrm{I}}{\mathrm{C}_{1}^{2}}$ inversiert, wobei wir den Leitwertkreis $K_{g}$ mit dem Mittelpunkte $M_{g}$ erhalten. Die direkte Konstruktion dieses Kreises, der als "Grundkreis des Kaskadendiagramms" bezeichnet werden möge, wird in den Abschnitten 6 und 7 gezeigt werden.

Die Punkte $P_{g}$ des Grundkreises entsprechen den Punkten $p_{\mathrm{g}}$ des Kreises $k_{g}$. Die durch die Punkte $p_{g}$ gezogenen Parallelen zur v'-Achse gehen bei der in Rede stehenden Inversion in Kreise über, welche durch die Punkte $P_{\mathrm{g}}$ des. Grundkreises und den Pol $\mathrm{O}^{\prime}$ gehen und deren Mittelpunkte $\mathrm{M}_{\mathrm{h}}$ auf der $\mathbf{u}^{\prime}$-Achse liegen. Auf diesen „Hilfskreisen" liegen daher auch die den Punkten p der $\left(z_{\mathrm{r}}^{*}+\mathcal{Z}_{\mathrm{s}}\right)$-Kurve entsprechenden Punkte $\mathrm{P}$ des Kaskadendiagramms.

Nach Abb. 7 ist

$$
\operatorname{tg} \Varangle\left(\mathrm{PO}^{\prime} \mathrm{u}^{\prime}\right)=\operatorname{tg} \Varangle\left(\mathrm{p} \mathrm{O}^{\prime} \mathrm{u}^{\prime}\right)=\frac{\mathrm{r}_{\mathrm{I}}^{*}+\mathrm{r}_{3^{\mathrm{s}}}^{*}}{\mathrm{x}_{\mathrm{I}}^{*}+\mathrm{X}_{2 \mathrm{~s}}}+\frac{\frac{\mathrm{r}_{2}}{\alpha \mathrm{s}+\beta}}{\mathrm{x}_{\mathrm{I}}^{*}+\mathrm{X}_{2 \mathrm{~s}}} ;
$$

da

ist äuch

$$
\operatorname{tg} \Varangle\left(\mathrm{P}_{\mathrm{g}} \mathrm{O}^{\prime} \mathrm{u}^{\prime}\right)=\operatorname{tg} \Varangle\left(\mathrm{p}_{\mathrm{g}} \mathrm{O}^{\prime} \mathrm{u}^{\prime}\right)=\frac{\mathrm{r}_{\mathrm{I}}^{*}+\mathrm{r}_{3 \mathrm{~s}}^{*}}{\mathrm{x}_{\mathrm{I}}^{*}+\mathrm{X}_{2 \mathrm{~s}}},
$$




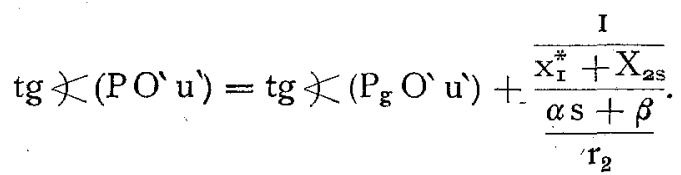

Wenn wir Zähler und Nenner des Bruchausdruckes mit dem Verhältnis $\frac{\mathrm{I}}{\mathrm{C}_{1}^{2}}$ multiplizieren und dann

setzen, wird

$$
\frac{\frac{\mathrm{I}}{\mathrm{C}_{1}^{2}} \cdot \frac{\mathrm{I}}{\mathrm{X}_{\mathrm{I}}^{*}+\mathrm{X}_{2 \mathrm{~s}}}}{\frac{\mathrm{I}}{\mathrm{C}_{1}^{2}} \cdot \frac{\alpha \mathrm{s}+\beta}{\mathrm{r}_{2}}}=\operatorname{tg} \psi_{\mathrm{s}}
$$

$$
\operatorname{tg} \Varangle\left(\mathrm{PO}^{\prime} \mathrm{u}^{\prime}\right)=\operatorname{tg} \Varangle\left(\mathrm{P}_{\mathrm{g}} \mathrm{O}^{\prime} \mathrm{u}^{\prime}\right)+\operatorname{tg} \psi_{\mathrm{s}} \text { : }
$$

In dieser Beziehung ist bei gegebenem Grundkreis $\operatorname{tg} \Varangle\left(\mathrm{P}_{\mathrm{g}} \mathrm{O}^{\prime} \mathrm{u}^{\prime}\right)$ bekannt, während sich $\operatorname{tg} \psi_{\mathrm{s}}$ nach 45 ) ermitteln läßt. Wie aus $\mathrm{Abb} .7 \mathrm{zu}$ ersehen ist, entspricht der Blindwiderstand $\mathrm{x}_{\mathbf{r}}^{*}+\mathrm{X}_{2 \mathrm{~s}}$ dem Abstand der durch die Punkte $\mathrm{p}_{\mathrm{g}}$ des Kreises $\mathrm{k}_{\mathrm{g}}$ gezogenen Parallelen zur $\mathrm{v}^{\prime}$-Achse vom Ursprung $\mathrm{O}^{\prime}$; es stellen daher die Blindleitwerte

$$
\frac{I}{C_{1}^{q}} \cdot \frac{I}{X_{I}^{*}+X_{2 s}}=2 R_{h}
$$

die Durchmesser der Hilfskreise dar, in welche diese Parallelen bei der mit Berücksichtigung des Reduktionsverhältnisses $\frac{\mathrm{I}}{\mathrm{C}_{1}^{2}}$ durchgeführten Inversion übergehen.

Wenn wir nun durch einen beliebigen Punkt $\mathrm{P}_{\mathrm{g}}$ des Grundkreises den Hilfskreis ziehen und seinen Halbmesser $R_{h}$ in dem der Schlüpfung $s$ des Punktes $P_{g}$ entsprechenden Abstand

$$
\frac{\mathrm{I}}{2 \mathrm{C}_{1}^{2}} \cdot \frac{\alpha \mathrm{S}+\beta}{\mathrm{r}_{2}}
$$

senkrecht zur $\mathrm{u}^{\prime}$-Achse auftragen, so ist die Verbindungsgerade - des erhaltenen Punktes $\psi$ mit $O^{\prime}$ nach 45) unter dem $\Varangle \psi_{\text {s }}$ gegen die $u^{\prime}$-Achse geneigt. Im gleichen Maßstabe mißt der Abschnitt $\pi_{\mathrm{g}} \mathrm{u}_{\mathrm{s}}^{\prime}$ dieser Senkrechten zwischen der Verbindungsgeraden $\mathrm{O}^{\prime} \mathrm{P}_{\mathrm{g}}$ und der $u^{\prime}$-Achse $\operatorname{tg} \Varangle\left(\mathrm{P}_{\mathbf{g}} \mathrm{O}^{\prime} \mathrm{u}^{\prime}\right)$. Tragen wir daher vom Punkte $\pi_{\mathrm{g}}$ den Halbmesser $\mathrm{R}_{\mathrm{h}}=\overline{\pi_{\mathrm{g}} \pi}$ des Hilfskreises auf, so ist die Strecke $\overline{\pi \mathrm{u}_{\mathrm{s}}}$ nach 46) $\operatorname{tg} \Varangle\left(\mathrm{PO}^{\prime} \mathrm{u}^{\prime}\right)$ proportional. Im Schnitte der Verbindungsgeraden $\mathrm{O}^{\prime} \pi$ mit dem Hilfskreis ergibt sich schließlich der der betreffenden Schlüpfung entsprechende Punkt $P$ des Kaskadendiagramms. - Die Punkte $u_{s}^{\prime}$ stellen auf der $u^{\prime}$-Achse eine lineare Schlüpfungsskala dar und lassen sich daher leicht eintragen.

5. Beziehungen für das Stromdiagramm der Kaskadenschaltung. Bevor wir auf die direkte Konstruktion des Grundkreises eingehen, wollen wir aus Abb. 7 einige wichtige Beziehungen für das Stromdiagramm der Kaskadenschaltung ableiten, welche die graphische Darstellung der Drehmomente ermöglichen.

Wenn die Scheinleitwerte $\mathfrak{Y}_{\mathrm{s}}$ mit der als konstant vorausgesetzten primären Klemmenspannung $\mathfrak{P}_{1}$ multipliziert werden, ergeben sich die primären Stromaufnahmen der Kaskadenschaltung; es kann aber auch direkt das Leitwertdiagramm als Stromdiagramm für konstante primäre Klemmenspannung angesehen werden, wenn die Strecken desselben in einem dieser Spannung entsprechenden Strommaßstabe gemessen werden. Die auf diesen Fall bezughabenden Größen sollen durch den unteren Zeiger „Strom" unterschieden werden. 
Im Stromdiagramm stellen die Vektoren

$$
\overline{\mathrm{OP}}_{\text {Strom }}=\widetilde{\Im}_{1}=\mathfrak{B}_{1} \mathfrak{⿰} \text { s }
$$

die Primärströme dar, welche mit dem in die Richtung der pos. v-Achse fallenden Vektor der primären Klemmenspannung die primären Phasenwinkel $\varphi_{1}=\Varangle(\mathrm{POV})$ einschließen. Die primäre elektrische Leistung der Kaskadenschaltung

$$
\mathrm{W}_{1}=\mathrm{P}_{1} \mathrm{I}_{1} \cos \varphi_{\mathrm{i}}
$$

wird durch die auf (uv) bezogenen Ordinaten

$$
\mathrm{v}(\mathrm{P})_{\text {Strom }}=\mathrm{I}_{1} \cos \varphi_{1}
$$

der Punkte $\mathrm{P}$ des Stromdiagramms im Maßstabe $\frac{\mathrm{W}_{1}}{\mathrm{P}_{1}}$ gemessen.

Es soll nun untersucht werden, welche Bedeutung die Vektoren

$$
\overline{\mathrm{O}}^{\prime} \mathrm{P}_{\text {Strom }}=\mathfrak{P}_{1} \mathfrak{Y}_{\mathrm{s}} \text { ( } \text { (siehe 26a) }
$$

im Stromdiagramm der Kaskadenschaltung besitzen.

Nach Abb. 5 a ist das Verhältnis zwischen dem Sekundär- und Primärstrome der Kaskadenschaltung

da nach 49) und 24)

$$
\frac{\widetilde{J}_{2}}{\widetilde{\Im}_{1-}}=\frac{\mathfrak{z}_{\mathrm{a}}}{\mathfrak{z}_{\mathrm{a}}+\mathfrak{Z}_{2 \mathrm{~s}}}
$$

wird

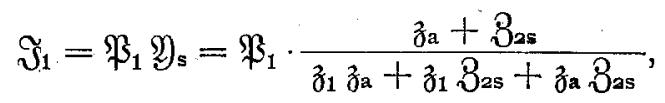

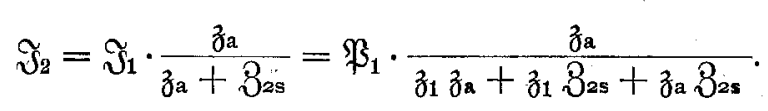

Der vorstehende Ausdruck kann nach Einführung des vektoriellen Verhältnisses $\widetilde{S}_{1}(27)$ und des Scheinwiderstandes ${ }_{0}^{*}$ (28) in der Form

$$
\widetilde{J}_{2}=\frac{\mathfrak{P}_{1}}{\mathfrak{S}_{1}} \cdot \frac{1}{\tilde{\partial}_{\mathrm{r}}^{*}+Z_{\mathrm{as}}}
$$

geschrieben werden; wenn wir hierin nach 26 a)

setzen, wird

$$
\frac{\mathrm{I}}{\mathfrak{E}_{1}} \cdot \frac{\mathrm{I}}{\tilde{z}_{\mathrm{I}}^{*}+Z_{2 \mathrm{~s}}}=\mathfrak{S}_{1} \mathfrak{Y}_{\mathrm{s}}^{\prime}
$$

$$
\Im_{2}=\mathfrak{P}_{1} \mathfrak{c}_{1} \mathfrak{V}_{\mathrm{s}}^{\prime} .
$$

Aus $52 \mathrm{~b}$ ) und 50) folgt die Beziehung

$$
{\overline{\mathrm{O}} \mathrm{N} \overline{\mathrm{P}}_{\mathrm{Strom}}}=\frac{\widetilde{\mathfrak{I}}_{2}}{\widetilde{\mathfrak{C}}_{1}}
$$

$\mathrm{Da}$ das vektorielle Verhältnis $\widetilde{\Xi}_{1}$ konstant ist, sind die Vektoren $\overline{\mathrm{O}^{\prime} \mathrm{P}}$ den Sekundärströmen $\mathfrak{J}_{2}$ der Kaskadenschaltung proportional.

Aus 52 a) ergibt sich der Betrag des Sekundärstromes zu

$$
\mathrm{I}_{2}=\frac{\mathrm{P}_{1}}{\mathrm{C}_{1}} \cdot \frac{\mathrm{I}}{\sqrt{\left(\mathrm{r}_{\mathrm{I}}^{*}+\mathrm{R}_{2 \mathrm{~s}}\right)^{2}+\left(\mathrm{x}_{\mathrm{I}}^{*}+\mathrm{X}_{2 \mathrm{~s}}\right)^{2}}} \text {. }
$$

Schließlich wollen wir noch untersuchen, welche Bedeutung die Abschnitte $\overline{\mathrm{Pa}}, \overline{\mathrm{ab}}$ und $\overline{\mathrm{Pb}}$ (siehe Abb. 7 ) der auf (u`v') bezogenen Ordinaten v'(P) der Punkte $P$ im Stromdiagramm der Kaskadenschaltung besitzen. Die Punkte a liegen auf den Verbindungsgeraden des Punktes $O^{\prime}$ mit den Fußpunkten $f_{3}$ der auf $\left(u_{3} v_{3}\right)$ bezogenen Ordinaten der Pünkte $p_{g}$ des Kreises $\dot{k}_{g}$, die Punkte $b$ auf den Verbindungsgeraden $\mathrm{O}^{\prime} \mathrm{P}_{\mathrm{g}}$. 
Nach Abb. 7 besteht die Proportion

Aus der Beziehung

$$
\overline{\mathrm{Pa}}_{\text {Strom }}:{\overline{\mathrm{O}^{\prime} \mathrm{P}}}_{\text {Strom }}=\overline{\mathrm{pf}}_{\mathbf{3}}\left(=\mathrm{R}_{2 \mathrm{~s}}\right):{\overline{\mathrm{O}^{\prime} \mathrm{p}}}
$$

ergibt sich

$$
\overline{\mathrm{O}}^{\prime} \mathrm{P}_{\mathrm{Strom}}=\frac{\mathrm{P}_{1}}{\mathrm{C}_{1}^{2}} \cdot \frac{\mathrm{I}}{\overline{\mathrm{O}^{\prime} \mathrm{p}}}=\frac{\mathrm{I}_{2}}{\mathrm{C}_{1}}(44 \text { und } 50 \mathrm{a})
$$

und damit

$$
\overline{\mathrm{O}^{\prime} \mathrm{p}}=\frac{\mathrm{P}_{1}}{\mathrm{C}_{1} \mathrm{I}_{2}}
$$

$$
\overline{\mathrm{Pa}}_{\text {Strom }}=\frac{\mathrm{I}_{2}^{2} \mathrm{R}_{\mathrm{as}}}{\mathrm{P}_{1}} \text {. }
$$

Für die Abschnitte $\overline{\mathrm{ab}}_{\text {Strom }}$ und $\overline{\mathrm{Pb}}_{\text {Strom }}$ ergeben sich auf eine ähnliche Weise die Beziehungen

$$
\overline{\mathrm{ab}}_{\text {Strom }}=\frac{\mathrm{I}_{2}^{2} \mathrm{r}_{3 \mathrm{~s}}^{*}}{\mathrm{P}_{1}}
$$

bzw.

$$
\overline{\mathrm{Pb}}_{\text {Strom }}=\frac{I_{2}^{2} \cdot \frac{\mathrm{r}_{2}}{\alpha \mathrm{s}+\beta}}{\mathrm{P}_{1}} .
$$

Die Abschnitte $\overline{\mathrm{Pa}}_{\text {Strom }}, \overline{\mathrm{ab}}_{\text {Strom }}$ und $\overline{\mathrm{Pb}}_{\text {Strom }}$ messen demnach im Stromdiagramm der Kaskadenschaltung die Leistungen $\mathrm{I}_{2}^{2} \mathrm{R}_{2 \mathrm{~s}}, \mathrm{I}_{2}^{2} \mathrm{r}_{3 \mathrm{~s}}^{*}$ bzw. $\mathrm{I}_{2}^{2} \cdot \frac{\mathrm{r}_{2}}{\alpha \mathrm{s}+\beta}$ in demselben Maßstabe wie die auf ( $\mathrm{u} v$ ) bezogenen Ordinaten $\mathrm{v}(\mathrm{P})$ Strom $\operatorname{der}$ Punkte $\mathrm{P}$ die primäre elektrische Leistung $W_{1}$; dieses Ergebnis wird bei der Darstellung der Drehmomente der Kaskadenschaltung (Abschnitt 8) verwendet werden.

\section{Direkte Konstruktion des Grundkreises.}

Wenn wir in 26a) für den zusammengefaßten Scheinwiderstand $\mathrm{B}_{\mathrm{s}}$ den Ausdruck 23) einführen und

setzen, erhalten wir

$$
z_{1}^{*}+z_{2 s}=z_{1 I s}
$$

$$
\mathfrak{Y}_{\mathrm{s}}^{\prime}=\frac{\mathrm{I}}{\mathbb{E}_{1}^{2}} \cdot \frac{z_{\mathrm{b}}+z_{3} \mathrm{~s}^{*}}{z_{I I s} z_{\mathrm{b}}+z_{I I s} z_{3} \mathrm{~s}+z_{\mathrm{b}} z_{3} \mathrm{~s}}
$$

mit Einführung des vektoriellen Verhältnisses

und des Scheinwiderstandes

$$
\mathfrak{D}_{\mathrm{II}}=\mathrm{D}_{\mathrm{II} \mathrm{s}} \mathrm{e}^{-\mathrm{j} \delta_{\mathrm{II}}}=\frac{z_{\mathrm{II} \mathrm{s}}+\xi_{\mathrm{b}}}{z_{\mathrm{b}}}
$$

$$
\mathfrak{z}_{\mathrm{II} s}^{*}=\frac{\mathfrak{Z I I ~}_{\mathrm{II}}}{\mathfrak{D}_{\mathrm{II}}}
$$

läßt sich $\mathfrak{V}_{\mathrm{s}}^{\prime}$ in ähnlicher Weise wie früher $\mathfrak{V}_{\mathrm{s}}$ (siehe 24) bis 28) und 24a) in zwei Summanden zerlegen:

$$
\mathfrak{Y}_{\mathrm{s}}=\frac{\mathrm{I}}{\mathfrak{C}_{1}^{2}} \cdot \frac{\mathrm{I}}{\partial \mathrm{IIs}+z_{\mathrm{b}}}+\frac{1}{\mathfrak{E}_{1}^{2}} \cdot \frac{\mathrm{I}}{\mathfrak{D}_{1 \mathrm{I}}^{2} \mathrm{~s}} \cdot \frac{\mathrm{I}}{z_{\mathrm{II}}^{*}+\mathfrak{z}_{3} \mathrm{~s}} .
$$

Der resultierende Scheinleitwert der Kaskadenschaltung ist dann

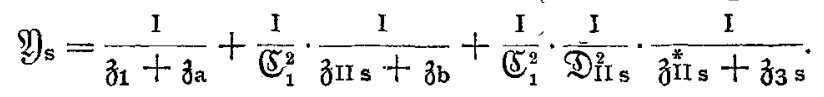

Für den Grundkreis ist $\frac{\mathrm{r}_{2}}{\alpha \mathrm{s}+\beta}=\mathrm{O}$; es sind daher die in 57) und 60) eingeführten Scheinwiderstände zils und $z_{11 s}^{*}$ sowie das vektorielle Verhältnis $\mathscr{D}_{\text {IIs }}(59)$ für den Grundkreis konstant, was durch Weglassung des Zeigers $s$ hervorgehoben

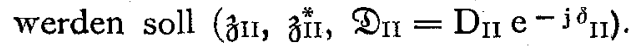


Wenn wir diese Größen in 62) einführen, erhalten wir den dem Grundkreis entsprechenden Scheinleitwert

$$
\mathfrak{V}_{\mathrm{s}}\left(\frac{\mathrm{r}_{2}}{\alpha \mathrm{s}+\beta}=0\right)=\frac{I}{z_{1}+z_{a}}+\frac{I}{\mathfrak{C}_{1}^{2}} \cdot \frac{I}{z_{I I}+z_{b}}+\frac{I}{\mathfrak{C}_{1}^{2}} \cdot \frac{I}{\mathfrak{D}_{I I}^{2}} \cdot \frac{I}{z_{I I}^{*}+z_{3 s}},
$$

welcher auch in der Form

$$
\mathfrak{V}_{\mathrm{s}}\left(\frac{r_{2}}{\alpha_{s}+\beta}=0\right)=\frac{I}{z_{1}+z_{2}}+\frac{I}{\mathrm{C}_{1}^{2}} \cdot \frac{I}{\left(z_{I I}+z_{b}\right) e^{-j_{1} \gamma_{1}}}+\frac{I}{C_{1}^{2}} \cdot \frac{I}{D_{I I}^{2}} \cdot \frac{I}{\left(z_{I I}^{*}+z_{3} s\right) e^{-j\left(a \gamma_{1}+2 \delta_{I I}\right.}} \cdot 6
$$

Diese Gleichung ermöglicht nun, wie in Abb. 8 gezeigt, die direkte Konstruktion des Grundkreises, auf dem in weiterer Folge das Kaskadendiagramm aufgebaut werden kann. Dabei wurden folgende Widerstands- und Polpaarzahlverhältnisse zugrunde gelegt:

$$
\begin{array}{lllll}
\mathrm{r}_{1}=0.8 & \mathrm{r}_{\mathrm{a}}=8 & \mathrm{r}_{2}=2 & & \mathrm{r}_{3 \mathrm{t}}=\mathrm{I} \cdot 2 \\
\mathrm{x}_{1}=\mathrm{I} .6 & \mathrm{x}_{\mathrm{a}}=40 & \mathrm{x}_{2}=4 & \mathrm{x}_{\mathrm{b}}=60 & \mathrm{x}_{3}=2.4 \\
\alpha=\frac{3}{5} & & & \\
\beta & =\frac{2}{5} . & & &
\end{array}
$$

Das erste Glied von 63 a) ist nach früherem der Scheinleitwert $\mathfrak{Y}\left(-\frac{\beta}{\alpha}\right)$ der Kaskadenschaltung für die Schlüpfung $s=-\frac{\beta}{\alpha}$, welche dem Fall entspricht, daß der Läufer der vorderen Maschine synchron mit ihrem Drehfelde rotiert. In dem Koordinatensystem (u v) der Abb. 8 wird dieser Leitwert durch die Strecke $\overline{O^{\circ}}$ dargestellt. Die auf (u v) bezogenen Koordinaten des Punktes $\mathrm{O}^{\prime} \equiv \mathrm{P}_{-} \frac{\beta}{\alpha}$ sind

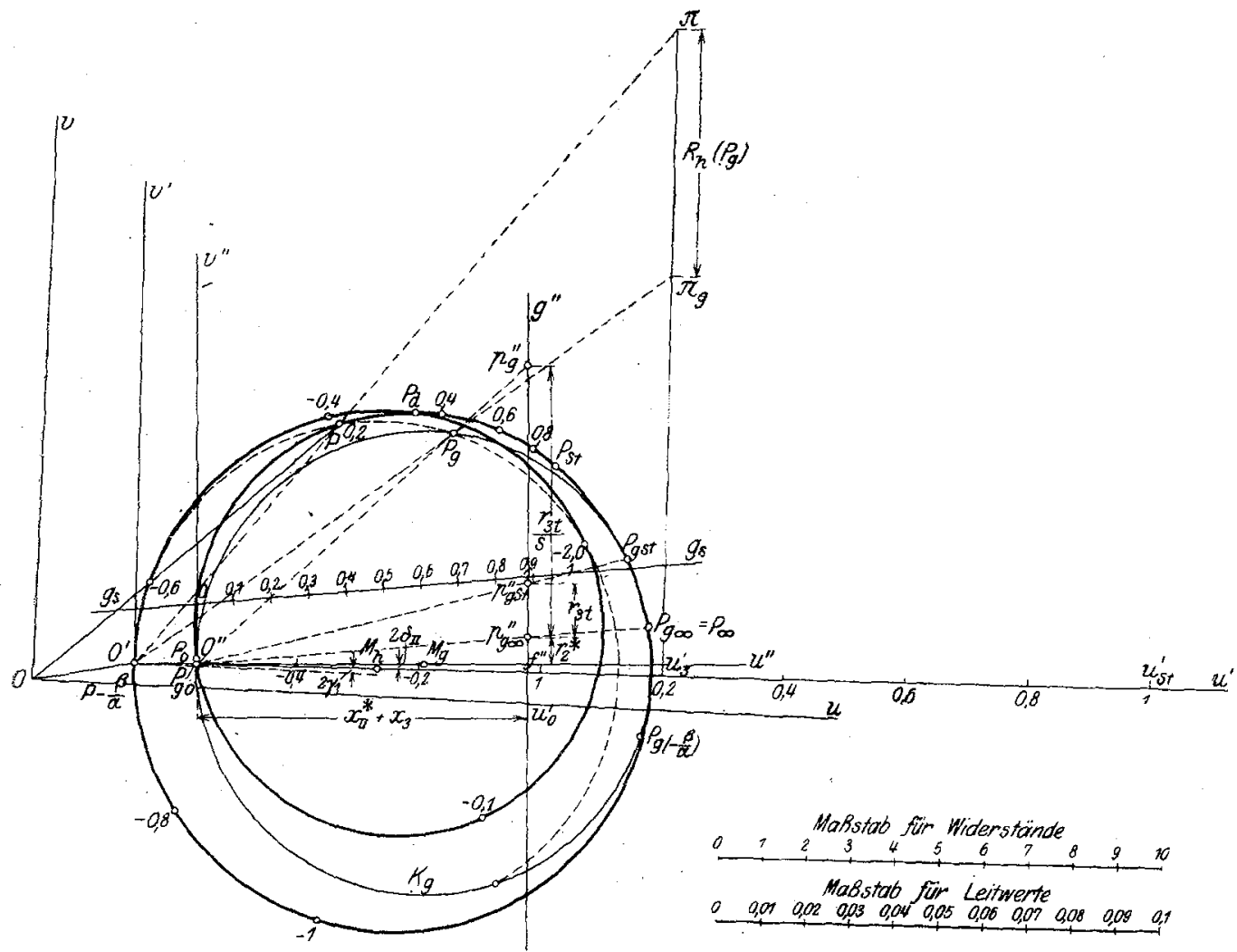

Abb. 8. 


$$
\begin{aligned}
& \mathrm{v}\left(\mathrm{O}^{\prime} \equiv \mathrm{P}_{-} \frac{\beta}{\alpha}\right)=\frac{\mathrm{r}_{1}+\mathrm{r}_{\mathrm{a}}}{\left(\mathrm{r}_{1}+\mathrm{r}_{\mathrm{a}}\right)^{2}+\left(\mathrm{x}_{1}+\mathrm{x}_{\mathrm{a}}\right)^{2}}=0,004 \underset{\text { (Leitwerteînheiten) }}{\mathrm{L} . \text { E. }} \\
& \mathrm{u}\left(\mathrm{O}^{\prime} \equiv \mathrm{P}_{-} \frac{\beta}{\alpha}\right)=\frac{\mathrm{x}_{1}+\mathrm{x}_{\mathrm{a}}}{\left(\mathrm{r}_{1}+\mathrm{r}_{\mathrm{a}}\right)^{2}+\left(\mathrm{x}_{\mathrm{t}}+\mathrm{x}_{\mathrm{a}}\right)^{2}}=0,023 \text { L. E. }
\end{aligned}
$$

Durch den Punkt $\mathrm{O}^{`} \equiv \mathrm{P}_{-}-\frac{\beta}{\alpha}$ als Ursprung legen wir das uns schon bekannte Achsensystem $\left(u^{\prime} v^{\prime}\right)$, welches gegen ( $\left.u v\right)$ um den Winkel $2 \gamma_{1}$ entgegen dem Uhrzeigersinne verdreht ist; der einfache Winkel $\gamma_{1}$ läßt sich aus dem nach 29) für

$$
\operatorname{tg} \gamma_{1}=\frac{\frac{x_{a}}{r_{a}}-\frac{x_{1}+x_{a}}{r_{1}+r_{a}}}{I+\frac{x_{a}}{r_{a}} \cdot \frac{x_{1}+x_{a}}{r_{1}+r_{a}}}=\frac{I}{90,9}
$$

errechneten Zahlenverhältnis ermitteln. Es bietet gewisse Vorteile, wenn die

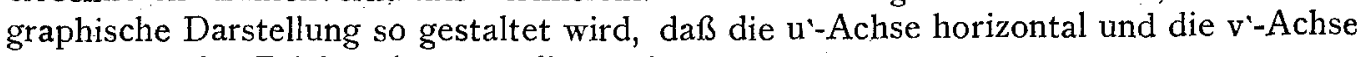
vertikal in die Zeichenebene zu liegen kommt.

Wenn wir den dem zweiten Gliede von 63a) entsprechenden Scheinleitwert

$$
\overline{\mathrm{O}^{\prime} \mathrm{O} "}=\frac{\mathrm{I}}{\mathrm{C}_{1}^{2}} \cdot \frac{\mathrm{I}}{\left(z_{\mathrm{II}}+z_{\mathrm{b}}\right) \mathrm{e}^{-\mathrm{j} a \gamma_{\mathrm{t}}}}
$$

auf ( $\left.u^{\prime} v^{\prime}\right)$ beziehen, entfällt das Drehglied $e^{-j a \gamma_{1}}$, so daß

$$
\overline{O^{\prime} O \cdot(a \cdot v)}=\frac{I}{C_{1}^{2}} \cdot \frac{I}{z_{I I}+z_{b}}=\frac{I}{C_{1}^{2}} \cdot \frac{I}{r_{1}^{*}+j\left(x_{1}^{*}+x_{2}+x_{b}\right)}
$$

dabei ist nach $27 \mathrm{~b}$ )

$$
C_{1}^{2}=\frac{\left(r_{1}+r_{a}\right)^{2}+\left(x_{1}+x_{a}\right)^{2}}{z_{a}^{2}}=I, 087
$$

nnd nach 3oa)

$$
\begin{aligned}
& r_{1}^{*}=\frac{I}{C_{1}^{2}} \cdot\left[r_{1}+r_{a}\left(\frac{z_{1}}{z_{a}}\right)^{2}\right]=0,75 \text { W. E. } \\
& x_{1}^{*}=\frac{I}{C_{1}^{2}} \cdot\left[x_{1}+x_{a}\left(\frac{z_{1}}{z_{a}}\right)^{2}\right]=I, 544 \text { W. E. }
\end{aligned}
$$

Aus 67) ergeben sich die auf ( $\left.\mathrm{u}^{\prime} \mathrm{v}^{\prime}\right)$ bezogenen Koordinaten des Punktes 'O' zu

$$
\begin{aligned}
& \mathrm{v}^{\prime}\left(\mathrm{O}^{\prime \prime}\right)=\frac{\mathrm{I}}{\mathrm{C}_{1}^{2}} \cdot \frac{\mathrm{r}_{1}^{*}}{\left(\mathrm{r}_{1}^{*}\right)^{2}+\left(\mathrm{x}_{1}^{*}+\mathrm{x}_{2}+\mathrm{x}_{\mathrm{b}}\right)^{2}}=0,000 \mathrm{r} 6 \mathrm{~L} . \mathrm{E} ., \\
& \mathrm{u}^{\prime}\left(\mathrm{O}^{\prime \prime}\right)=\frac{\mathrm{I}}{\mathrm{C}_{1}^{2}} \cdot \frac{\mathrm{x}_{1}^{*}+\mathrm{x}_{2}+\mathrm{x}_{\mathrm{b}}}{\left(\mathrm{r}_{1}^{*}\right)^{2}+\left(\mathrm{x}_{1}^{*}+\mathrm{x}_{2}+\mathrm{x}_{\mathrm{b}}\right)^{2}}=0,0 \mathrm{I}_{4} \mathrm{~L} . \mathrm{E} .
\end{aligned}
$$
leitwertes

$$
\mathfrak{Y}_{s} \cdot\left(\frac{\mathrm{r}_{2}}{\alpha \mathrm{s}+\bar{\beta}}=0\right)=\frac{\mathrm{I}}{\mathrm{C}_{1}^{2}} \cdot \frac{\mathrm{I}}{\mathrm{D}_{\mathrm{II}}^{2}} \cdot \frac{\mathrm{I}}{\left(\mathrm{z}_{\mathrm{g}}^{\mathrm{z}}+z_{3}\right) \mathrm{e}^{-\mathrm{j}\left(2 \gamma_{1}+2 \delta_{\mathrm{II}}\right)}}
$$

kann durch Inversion aus dem geometrischen Orte des Scheinwiderstandes $\left(z_{I I}^{*}+z_{3} s\right) e^{-j\left(2 \gamma_{1}+2 \delta_{I I}\right)}$ abgeleitet werden, wobei die erhaltenen Scheinleitwerte im Verhältnis $\frac{\mathrm{I}}{\mathrm{C}_{1}^{2}} \cdot \frac{\mathrm{I}}{\mathrm{D}_{\mathrm{II}}^{2}}$ zu reduzieren sind.

Das vektorielle Verhältnis

$$
\mathfrak{D}_{\mathrm{II}}=\mathrm{D}_{\mathrm{II}} \mathrm{e}^{-\mathrm{j} \ddot{o}_{\mathrm{II}}}=\frac{z_{\mathrm{II}}+z_{\mathrm{b}}}{z_{\mathrm{b}}}
$$


wird durch die Scheinwiderstände $z_{I I}+z_{b}$ (wobei $z_{I I}=z_{1}^{*}+j x_{2}$ ) und $z_{b}=j x_{b}$ in ähnlicher Weise bestimmt, wie $\mathscr{E}_{1}=\mathrm{C}_{1} \mathrm{e}^{-\mathrm{j} \gamma_{1}}$ durch $\left(z_{1}+z_{\mathrm{a}}\right)$ und $z_{\mathrm{a}} ;$ desgleichen besteht zwischen den Scheinwiderständen

$$
z_{\mathrm{II}}^{*}=\frac{z_{\mathrm{II}}}{\mathfrak{D}_{\mathrm{II}}}
$$

und $z_{1}^{*}=\frac{z_{1}}{\mathfrak{C}_{1}}$ eine Analogie. Dementsprechend ergeben sich die auf diese Größen bezughabenden Ausdrücke

$$
\begin{aligned}
& \operatorname{tg} \delta_{\mathrm{II}}=\frac{\mathrm{r}_{1}^{*}}{\mathrm{x}_{1}^{*}+\mathrm{x}_{2}+\mathrm{x}_{\mathrm{b}}}=\frac{\mathrm{I}}{87,3} \\
& \mathrm{D}_{\mathrm{II}}^{2}=\frac{\left(\mathrm{r}_{1}^{*}\right)^{2}+\left(\mathrm{x}_{1}^{*}+\mathrm{x}_{\mathrm{2}}+\mathrm{x}_{\mathrm{b}}\right)^{2}}{\mathrm{x}_{\mathrm{b}}^{2}}=\mathrm{I}, \mathrm{I} 93 \\
& r_{I I}^{*}=\frac{r_{1}^{*}}{D_{I I}^{2}}=0.629 \text { W. E., } \\
& \mathrm{x}_{1 \mathrm{I}}^{*}=\frac{\mathrm{I}}{\mathrm{D}_{\mathrm{II}}^{2}} \cdot\left[\mathrm{x}_{1}^{*}+\mathrm{x}_{2}+\mathrm{x}_{\mathrm{b}}\left(\frac{z_{\mathrm{II}}}{\mathrm{x}_{\mathrm{b}}}\right)^{2}\right]=5,085 \text { W. E. ; } \mathrm{z}_{1 \mathrm{i}}^{2}=\left(\mathrm{r}_{\mathrm{x}}^{*}\right)^{2}+\left(\mathrm{x}_{1}^{*}+\mathrm{x}_{2}\right)^{2}
\end{aligned}
$$

Wir führen nun in Abb. 8 ein neues Koordinatensystem (u"v") mit O" als Ursprung ein, welches gegen ( $\left.u^{\prime} v^{\prime}\right)$ um den Winkel $2 \delta_{\text {II }}$, gegen (uv) daher um den Winkel $\left(2 \gamma_{1}+2 \delta_{\mathrm{II}}\right)$ entgegen dem Uhrzeigersinne verdreht ist, und tragen in dasselbe die gespiegelten Scheinwiderstände

$$
\left(z_{I I}^{*}+z_{3 s}\right)=r_{I I}^{*}+\frac{r_{3 t}}{s}+j\left(x_{I I}^{*}+x_{3}\right)
$$

ein; auf (uv) bezogen, stellen diese Vektoren die gespiegelten Scheinwiderstände

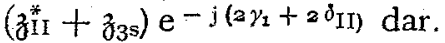

Bei veränderlicher Schlüpfung s beschreibt der Endpunkt $\mathrm{p}_{\mathbf{g}}^{\prime}$ ' der genannten Vektoren eine Gerade g", welche im Abstand

$$
\overline{\mathrm{O}^{\prime \prime} \mathrm{f}^{\prime \prime}}=\mathrm{x}_{\mathrm{II}}^{*}+\mathrm{x}_{3}=7,485 \mathrm{~W} . \mathrm{E} \text {. }
$$

von $\mathrm{O}^{\prime \prime}$ paralleI zur $v^{\prime \prime}$-Achse verläuft. Die den einzelnen Schlüpfungswerten entsprechenden Punkte dieser Geraden werden durch Auftragen der Strecken

$$
\overline{\mathrm{f}^{\prime \prime} \mathrm{p}_{\mathrm{g}}^{\prime \prime}}+\overline{\mathrm{p}_{\mathrm{g}} \mathrm{p}_{\mathrm{g}}^{\prime \prime}}=\mathrm{r}_{\mathrm{II}}^{*}+\frac{\mathrm{r}_{3 \mathrm{t}}}{\mathrm{s}}
$$

erhalten. Für $s=0$ wird $\frac{r_{3 t}}{s}=\infty$; diesem Schlüpfungswert entspricht der unendlich ferne Punkt der Geraden g". Der der Schlüpfung s=I (Stillstand) entsprechende Punkt $\mathrm{p}_{\mathrm{g}}$ st ergibt sich durch Auftragen der Strecke

$$
\overline{\mathrm{p}_{\mathrm{g} \infty}^{\prime \prime} \mathrm{p}_{\mathrm{g}}^{\prime \prime S t}}=\mathrm{r}_{3 \mathrm{t}}=\mathrm{I} \cdot 2 \text { W. E.; }
$$

der Punkt $p_{g}^{\prime}$ selbst entspricht den Schlüpfnngswerten $\pm \infty$, da hiefür $\frac{r_{3 t}}{s}=0$ wird.

Durch Inversion der im Abstande $x_{I I}^{*}+x_{3}$ von $O^{\prime \prime}$ parallel zur v"-Achse verlaufenden Geraden g" in bezug auf den Pol O" ergibt sich bei dem zu berücksichtigenden Reduktionsverhältnis $\frac{\mathrm{I}}{\mathrm{C}_{1}^{2}} \times \frac{1}{\mathrm{D}_{\mathrm{II}}^{2}}$ ein Kreis mit dem Durchmesser

$$
2 \mathrm{R}_{\mathrm{g}}=\frac{\mathrm{I}}{\mathrm{C}_{1}^{2}} \cdot \frac{\mathrm{I}}{\mathrm{D}_{\mathrm{II}}^{2}} \cdot \frac{\mathrm{I}}{\mathrm{x}_{\mathrm{II}}^{*}+\mathrm{x}_{3}}=0,1032 \mathrm{~L} . \mathrm{E} .
$$

welcher durch den Pol O" geht und dessen Mittelpunkt $\mathrm{M}_{\mathrm{g}}$ auf der u"-Achse liegt Dieser Kreis stellt, auf (u v) bezogen, den geometrischen Ort der Scheinleitwerte $\mathfrak{Y}_{\mathrm{s}}\left(\frac{r_{\mathbf{z}}}{\alpha s+\beta}=0\right)$ und damit den gesuchten Grundkreis $K_{g}$ des Kaskadendiagramms 
dar. Die Verbindungsgeraden $\overline{\mathrm{O}^{\prime \prime} \mathrm{p}_{\mathrm{g}}}$ schneiden den Kreis in Punkten $\mathrm{P}_{\mathbf{g}}$, wobei die Vektoren $\overline{\mathrm{O}^{\prime \prime} \mathrm{P}_{\mathrm{g}}}$, auf (uv) bezogen, die den Schlüpfungswerten der Punkte $\mathrm{P}_{\mathrm{g}}$ entsprechenden Scheinleitwerte $\mathfrak{Y}_{\mathrm{s}}^{\prime \prime}\left(\frac{\mathrm{r}_{2}}{\alpha_{\mathrm{s}+\beta}}=0\right)$ darstellen. Für die besonderen Schlüpfungswerte $\mathrm{s}=0$, I und $\pm \infty$ ergeben sich auf diese Weise die Kreispunkte $\mathrm{P}_{\mathrm{go}} \equiv \mathrm{O}^{\prime \prime}, \mathrm{P}_{\mathrm{gSt}}$ und $\mathrm{P}_{\mathrm{g} \infty}$, aus welchen sich mit Hilfe der in Abb. 8 gezeichneten Schlüpfungsgeraden $g_{s}$ die allen anderen Schlüpfungswerten entsprechenden Kreispunkte ermitteln lassen.

Werden die Leitwertvektoren $\overline{\mathrm{O}^{\prime \prime} \mathrm{P}_{\mathrm{g}}}$ auf das gegen (uv) um den Winkel $2 \gamma_{1}+2 \delta_{\text {II }}$ entgegen dem Uhrzeigersinne verdrehte Koordinatensystem (u" $v$ ") bezogen, so entfällt das Drehglied $e^{-j\left(2 \gamma_{1}+2 \delta_{I I}\right)}$ in $\left.7 \mathrm{I}\right)$ und es ist

$$
{\overline{\mathrm{O}^{\prime \prime}}}_{\mathrm{g}(\mathrm{u} \cdot \mathrm{v} \cdot)}=\frac{\mathrm{I}}{\mathrm{C}_{1}^{2}} \cdot \frac{\mathrm{I}}{\mathrm{D}_{\mathrm{II}}^{2}} \cdot \frac{\mathrm{I}}{\mathrm{z}_{\mathrm{II}}^{*}+\mathrm{z}_{3 \mathrm{~s}}}=\frac{\mathrm{I}}{\mathrm{C}_{1}^{2}} \cdot \frac{\mathrm{I}}{\mathrm{D}_{\mathrm{II}}^{2}} \cdot \frac{\mathrm{I}}{\left(\mathrm{r}_{\mathrm{II}}^{*}+\frac{\mathrm{r}_{3 \mathrm{t}}}{\mathrm{s}}\right)+\mathrm{j}\left(\mathrm{x}_{\mathrm{II}}^{*}+\mathrm{x}_{3}\right)} ;
$$

aus dieser Gleichung ergeben sich die auf (u" $v ")$ bezogenen Koordinaten der Punkte $\mathrm{P}_{\mathrm{g}}$ des Grundkreises zu

$$
\begin{aligned}
& \mathrm{v}^{\prime \prime}\left(\mathrm{P}_{\mathrm{g}}\right)=\frac{\mathrm{I}}{\mathrm{C}_{1}^{2}} \cdot \frac{\mathrm{I}}{\mathrm{D}_{\mathrm{II}}^{2}} \cdot \frac{\mathrm{r}_{\mathrm{II}}^{*}+\frac{\mathrm{r}_{3 \mathrm{t}}}{\mathrm{s}}}{\left(\mathrm{r}_{\mathrm{II}}^{*}+\frac{\left.\mathrm{r}_{3 \mathrm{t}}\right)^{2}+\left(\mathrm{x}_{\mathrm{II}}^{*}+\mathrm{x}_{3}\right)^{2}}{\mathrm{~s}}\right.}, \\
& \mathrm{u}^{\prime \prime}\left(\mathrm{P}_{\mathrm{g}}\right)=\frac{\mathrm{I}}{\mathrm{C}_{1}^{2}} \cdot \frac{\mathrm{I}}{\mathrm{D}_{\mathrm{II}}^{2}} \cdot \frac{\mathrm{x}_{\mathrm{II}}^{*}+\mathrm{x}_{3}}{\left(\mathrm{r}_{\mathrm{II}}^{*}+\frac{\mathrm{r}_{3 \mathrm{t}}}{\mathrm{s}}\right)^{2}+\left(\mathrm{x}_{\mathrm{II}}^{*}+\mathrm{x}_{3}\right)^{2}} .
\end{aligned}
$$

Für $\mathrm{s}=\mathrm{I}$ bžw. $\mathrm{s}= \pm \infty$ resultieren aus diesen Ausdrücken die auf (u"v") bezogenen Koordinaten der Punkte $\mathrm{P}_{\mathrm{g} \text { st }}$ und $\mathrm{P}_{\mathrm{g} \infty}$ des Grundkreises.

\section{Konstruktion des Kaskadendiagramms.}

Auf dem Grundkreise $K_{\mathrm{g}}$ läßt sich nach dem im Abschnitt 4 angegebenen Verfahren das Kaskadendiagramm punktweise aufbauen. Wir tragen zu diesem Behuf auf der 'u'-Achse die lineare Schlüpfungsskala

$$
\overline{\mathrm{O}^{\prime} \mathrm{u}_{\mathrm{s}}^{\prime}}=\frac{\mathrm{I}}{2 \mathrm{C}_{1}^{2}} \cdot \frac{\alpha \mathrm{s}+\beta}{\mathrm{r}_{2}}
$$

auf. Dem Schlüpfungswert $\mathrm{s}=0$ entspricht der Abstand

$$
\overline{\mathrm{O}^{\prime} \bar{u}_{0}}=\frac{\mathrm{I}}{2 \mathrm{C}_{1}^{2}} \cdot \frac{\beta}{\mathrm{r}_{2}}=0,092 \mathrm{I} \mathrm{L.} \mathrm{E.,}
$$

der Schlüpfung $s=I$ der Abstand

$$
\overline{\mathrm{O}^{\prime} \mathrm{u}_{\mathrm{St}}^{\prime}}=\frac{\mathrm{I}}{2 \mathrm{C}_{1}^{2}} \cdot \frac{\mathrm{I}}{\mathrm{r}_{2}}=0,23 \mathrm{~L} . \mathrm{E} \text {. }
$$

Für $\mathrm{s}=-\frac{\beta}{\alpha}=-\frac{2}{3}$ wird $\alpha \mathrm{s}+\beta=0$; der diesem Werte entsprechende Punkt der Schlüpfungsskala fällt mit $O^{\prime}$ zusammen.

Um aus einem beliebigen Punkte $P_{g}$ des Grundkreises, welcher dem auf der Geraden $g_{s}$ abzulesenden Schlüpfungswert s entspricht, den Punkt $P$ des Kaskadendiagramms für die gleiche Schlüpfung abzuleiten, gehen wir folgendermaßen vor. Wir ziehen den durch $\mathrm{P}_{\mathrm{g}}$ bestimmten Hilfskreis, welcher durch den Punkt O" geht und dessen Mittelpunkt $\mathrm{M}_{\mathrm{h}}$ auf der $\mathrm{u}^{\prime}$-Achse liegt; ferner errichten wir in dem der Schlüpfung s entsprechenden Punkt $u_{s}^{\prime}$ der Schlüpfungsskala eine Senkrechte zur $\mathrm{u}^{\prime}$-Achse und bringen sie mit der Verbindungsgeraden $\mathrm{O}^{\prime} \mathrm{P}_{\mathrm{g}}$ zum Schnitte. Von 
dem Schnittpunkte $\pi_{\mathrm{g}}$ tragen wir schließlich den Halbmesser $\mathrm{R}_{\mathrm{h}}\left(\mathrm{P}_{\mathrm{g}}\right)=\overline{\pi_{\mathrm{g}} \pi}$ des früher gezeichneten Hilfskreises auf; es schneidet dann die Verbindungsgerade $O^{\prime} \pi$ den Hilfskreis in dem gesuchten Punkte $P$ des Kaskadendiagramms. Die Halbmesser der Hilfskreise sind von den Punkten $\pi_{\mathrm{g}}$ stets nach aufwärts aufzutragen. Für einzelne Punkte wird eine proportionale Verkleinerung oder Vergrößerung der angegebenen Konstruktion erforderlich sein.

In $\mathrm{Abb} .8$ ist $\mathrm{P}_{\mathrm{o}}$ der Synchronismuspunkt $(\mathrm{s}=0)$ und $\mathrm{P}_{\mathrm{St}}$ der Stillstandspunkt $(s=\mathrm{I})$ des Kaskadendiagramms. Der den Schlüpfungswerten $\mathrm{s}= \pm \infty$ entsprechende Punkl $P_{\infty}$ desselben fällt mit dem Punkte $P_{g \infty}$ des Grundkreises zusammen. Der Punkt $\mathrm{O}^{\prime} \equiv \mathrm{P}_{-\frac{\beta}{\alpha}}$ entspricht nach früherem der Schlüpfung $\mathrm{s}=-\frac{\beta}{\alpha}$.

Da die Hilfskreise, den Grundkreis $K_{\mathrm{g}}$ in je zwei Punkten schneiden, welche den Schlüpfungswerten $\pm s$ entsprechen, liegen auch je zwei Punkte des Kaskadendiagramms auf einem Hilfskreise. Dabei ist zu beachten, daß in Abb. 8

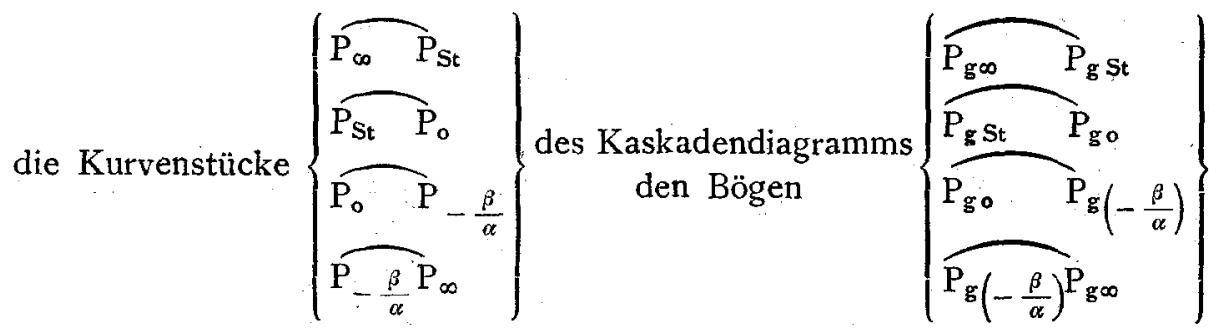

des Grundkreises entsprechen. Diesem Umstand ist bei der Konstruktion des Diagramms eine besondere Aufmerksamkeit zu widmen, um Verwechslungen zu vermeiden.

Geometrisch betrachtet stellt das Kaskadendiagramm elne Kurve vierter Ordnung dar. Sein . Doppelpunkt $P_{d}$ entspricht dem Doppelpunkt $p_{d}$ der $\left(a_{1}^{*}+B_{2 s}\right)$-Kurve in Abb. 7. Für die beiden Schlüpfungswerte im Doppelpunkt ergeben sich bei den der Abb. 8 zugrundegelegten Widerstands- und Polpaarzahlverhältriissen aus 43) die Werte

$$
s_{\mathrm{d}}= \pm \sqrt{\frac{\frac{\beta^{2}}{\alpha} \cdot \mathrm{x}_{\mathrm{b}}^{2}-\mathrm{r}_{2} \mathrm{r}_{3 \mathrm{t}}}{\alpha \mathrm{x}_{\mathrm{b}}^{2}+\frac{\mathrm{r}_{2}}{\mathrm{r}_{3 \mathrm{t}}} \cdot\left(\mathrm{x}_{\mathrm{b}}+\mathrm{x}_{3}\right)^{2}}}= \pm 0,333
$$

welche die Bestimmung des Doppelpunktes ermöglichen.

Wenn die Vektoren $\overline{O^{\prime} \mathrm{P}}$ auf das gegen (uv) um den Winkel $2 \gamma_{\mathrm{l}}$ entgegen dem Uhrzeigersinne verdrehte Koordinatensystem ( $\left.u^{\prime} v^{\prime}\right)$ bezogen werden, entfällt das Drehglied $e^{-j 2 \gamma_{2}}$ in 32) und es ist

$$
\overline{\mathrm{O}}^{\prime} \mathrm{P}_{(\mathrm{u} \cdot \mathrm{v})}=\frac{\mathrm{I}}{\mathrm{C}_{1}^{2}} \cdot \frac{\mathrm{I}}{\mathrm{z}_{1}^{*}+\mathrm{B}_{2 \mathrm{~s}}}=\frac{\mathrm{I}}{\mathrm{C}_{1}^{2}} \cdot \frac{\mathrm{I}}{\left(\mathrm{r}_{1}^{*}+\mathrm{R}_{2 \mathrm{~s}}\right)+\mathrm{j}\left(\mathrm{x}_{1}^{*}+\mathrm{X}_{2 \mathrm{~s}}\right)} .
$$

Aus dieser Gleichung ergeben sich die auf ( $u$ 'v') bezogenen Koordinaten der Punkte $\mathrm{P} z u$

$$
\begin{aligned}
& v^{\prime}(P)=\frac{I}{C_{1}^{2}} \cdot \frac{r_{I}^{*}+R_{2 s}}{\left(r_{I}^{*}+R_{2 s}\right)^{2}+\left(x_{I}^{*}+X_{2 s}\right)^{2}}, \\
& u^{\prime}(P)=\frac{1}{C_{1}^{2}} \cdot \frac{x_{I}^{*}+X_{2 s}}{\left(r_{I}^{*}+R_{2 s}\right)^{2}+\left(x_{I}^{*}+X_{2 s}\right)^{2}} .
\end{aligned}
$$


$R_{2 s}$ und $X_{2 s}$ sind die Komponenten des Scheinwiderstandes $Z_{2 s}$, welcher sich nach 23) aus den Scheinwiderständen

und

$$
z_{2 s}=\frac{r_{2}}{\alpha s+\beta}+j x_{2}
$$

$$
z_{3 s}^{*}=r_{3 s}^{*}+j x_{3 s}^{*}=\frac{z_{b} z_{3 s}}{z_{b}+z_{3 s}}
$$

zusammensetzt; letzterer läßt sich nach Einführung des mit der Schlüpfung veränderlichen vektoriellen Verhältnisses

auch in der Form

$$
\mathfrak{D}_{3 \mathrm{~s}}=\frac{\mathfrak{z}_{\mathrm{b}}+\mathfrak{z}_{3 \mathrm{~s}}}{z_{\mathrm{b}}}
$$

$$
z_{3 \mathrm{~s}}^{*}=\frac{\mathfrak{z}_{3 \mathrm{~s}}}{\mathfrak{D}_{3 \mathrm{~s}}}
$$

schreiben. Die Kompònenten von $z_{3}^{*}$ sind

wobei

$$
\begin{aligned}
& \mathrm{r}_{3 \mathrm{~s}}^{*}=\frac{\mathrm{I}}{\mathrm{D}_{3 \mathrm{~s}}^{2}} \cdot \frac{\mathrm{r}_{3 \mathrm{t}}}{\mathrm{s}}, \\
& \mathrm{x}_{3 \mathrm{~s}}^{*}=\frac{\mathrm{I}}{\mathrm{D}_{3 \mathrm{~s}}^{2}} \cdot\left[\mathrm{x}_{\mathrm{b}}\left(\frac{\mathrm{z}_{3 \mathrm{~s}}}{\mathrm{x}_{\mathrm{b}}}\right)^{2}+\mathrm{x}_{3}\right] ; \mathrm{z}_{3 \mathrm{~s}}^{2}=\left(\frac{\mathrm{r}_{3 \mathrm{t}}}{\mathrm{s}}\right)^{2}+\mathrm{x}_{3}^{2},
\end{aligned}
$$

$$
D_{3 \mathrm{~s}}^{2}=\frac{\left(\frac{r_{3 t}}{s}\right)^{2}+\left(x_{b}+x_{3}\right)^{2}}{x_{b}^{2}}
$$

das Quadrat des Betrages von $\mathfrak{D}_{3 \mathrm{~s}}$ bedeutet.

Es ist demnach

$$
\begin{aligned}
& \mathrm{R}_{2 \mathrm{~s}}=\frac{\mathrm{r}_{2}}{\alpha \mathrm{s}+\beta}+\mathrm{r}_{3 \mathrm{~s}}^{*}=\frac{\mathrm{r}_{2}}{\alpha \mathrm{s}+\beta}+\frac{\mathrm{r}}{\mathrm{D}_{3 \mathrm{~s}}^{2}} \cdot \frac{\mathrm{r}_{3 \mathrm{t}}}{\mathrm{s}} \\
& \mathrm{X}_{2 \mathrm{~s}}=\mathrm{x}_{2}+\mathrm{x}_{3 \mathrm{~s}}^{*}=\mathrm{x}_{2}+\frac{\mathrm{I}}{\mathrm{D}_{3 \mathrm{~s}}^{2}} \cdot\left[\mathrm{x}_{\mathrm{b}}\left(\frac{\mathrm{z}_{3 \mathrm{~s}}}{\mathrm{x}_{\mathrm{b}}}\right)^{2}+\mathrm{x}_{3}\right] .
\end{aligned}
$$

\section{Anderes Verfahren für die Ermittlung des Grundkreises.}

Der Grundkreis $\mathrm{K}_{\mathrm{g}}$ des Kaskadendiagramms läßt sich auch auf eine andere Weise ermitteln, welche in Abb. 9 dargestellt ist. Wir erinnern uns, daß der Kreis $\mathrm{K}_{\mathrm{g}}$ durch Inversion aus dem in Abb. 7 gezeichneten Widerstandskreise $\mathrm{k}_{\mathrm{g}}$ abgeleitet werden kann, wobei das Reduktionsverhältnis $\frac{\mathrm{I}}{\mathrm{C}_{1}^{2}} \mathrm{zu}$ berücksichtigen ist (siehe Abschnitt 4). Wir wollen nun zunächst die beiden Tangenten des Kreises $\mathrm{k}_{\mathrm{g}}$ parallel zur v'-Achse samt ihren Berührungspunkten $\mathrm{p}_{g}$, und $\mathrm{p}_{\mathrm{g} \infty}$ inversieren, welche nach Abb. 7 von $O^{\prime}$ die Abstände

bzw.

$$
u^{\prime}\left(\mathrm{pg}_{0}\right)=\mathrm{x}_{\mathrm{I}}^{\mathrm{*}}+\mathrm{x}_{2}+\mathrm{x}_{\mathrm{b}}
$$

$$
\mathrm{u}^{\prime}\left(\mathrm{p}_{\mathrm{g} \infty}\right)=\mathrm{u}^{\prime}\left(\mathrm{p}_{\mathrm{g} 0}\right)-2 \mathrm{r}_{\mathrm{g}}=\mathrm{x}_{\mathrm{I}}^{*}+\mathrm{x}_{\mathrm{g}}+\frac{\mathrm{x}_{\mathrm{b}} \mathrm{x}_{\mathrm{3}}}{\mathrm{x}_{\mathrm{b}}+\mathrm{x}_{\mathrm{3}}}
$$

haben. Die Berührungspunkte $p_{\mathbf{g}_{0}}$ und $\mathrm{p}_{\mathrm{g} \infty}$ liegen auf der $\mathrm{u}_{3}$-Achse, welche im Abstande

parallel zur u’-Achse verläuft.

$$
v^{\prime}\left(m_{g}\right)=r_{1}^{*}
$$


Bei der Inversion in bezug auf den Pol O' gehen die parallel zur v'-Achse gezogenen Tangenten des Kreises $\mathrm{k}_{\mathrm{g}}$ in die den Grundkreis berührenden Hilfskreise $\mathrm{K}_{\mathrm{h}_{0}}$ und $\mathrm{K}_{\mathrm{h}_{\infty}}$ über, welche durch den Pol $\mathrm{O}^{\prime}$ gehen und deren Mittelpunkte $\mathrm{M}_{\mathrm{b}_{0}}$ und $M_{h \infty}$ auf der u'-Achse liegen. Ihre Durchmesser ergeben sich aus den Abständen $\mathrm{u}^{\prime}\left(\mathrm{p}_{\mathrm{g} 0}\right)$ und $\mathrm{u}^{\prime}\left(\mathrm{p}_{\mathrm{g} \infty}\right) \mathrm{zu}$

bzw.

$$
2 R_{h 0}=\frac{I}{C_{1}^{2}} \cdot \frac{1}{x_{x}^{*}+x_{2}+x_{b}}
$$

$$
2 \mathrm{R}_{\mathrm{h} \infty}=\frac{I}{\mathrm{C}_{1}^{2}} \cdot \frac{\mathrm{I}}{\mathrm{x}_{\mathrm{I}}^{*}+\mathrm{x}_{2}+\frac{\mathrm{x}_{\mathrm{b}} \mathrm{x}_{3}}{\mathrm{x}_{\mathrm{b}}+\mathrm{x}_{3}}}
$$

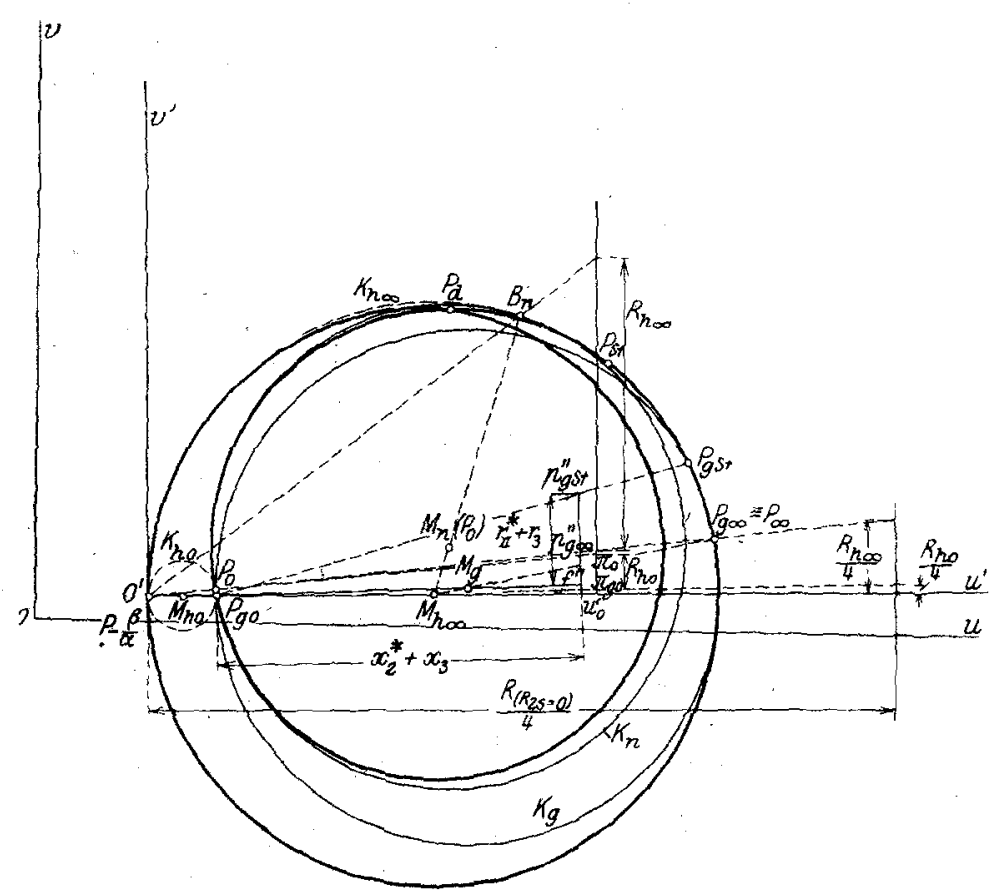

Abb. 9 .

Der Hilfskreis $\mathrm{K}_{\mathrm{h}_{0}}$ hat den kleinsten, $\mathrm{K}_{\mathrm{h}_{\infty}}$ den größten Durchmesser; diese Kreise schließen daher die Kaskadenkurve vollständig ein.

Die $\mathbf{u}_{3}$-Achse, deren Schnittpunkte mit der $\left(z_{i}^{*}+\beta_{2 s}\right)$-Kurve den Werten

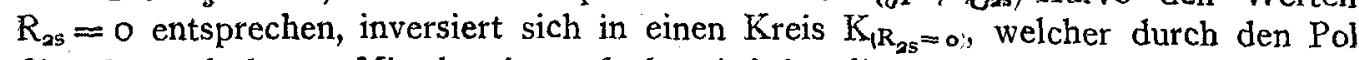
$O^{\prime}$ geht und dessen Mittelpunkt auf der $v^{\prime}$-Achse liegt; sein Durchmesser ergibt sich aus dem Abstande v' $\left(\mathrm{m}_{\mathrm{g}}\right)$ zu

$$
2 \mathrm{R}_{\left(\mathrm{R}_{2 \mathrm{~s}}=0\right)}=\frac{\mathrm{I}}{\mathrm{C}_{1}^{2}} \cdot \frac{\mathrm{I}}{\mathrm{r}_{\mathrm{I}}^{*}} \text {. }
$$

Dieser Kreis schneidet die Hilfskreise $K_{h o}$ und $K_{h_{\infty}}$ in den Punkten $P_{g_{0}}$ und $P_{g \infty}$ des Grundkreises, welche inversionsgemäß den Punkten $p_{g \circ}$ und $p_{g \infty}$ des Kreises $k_{g}$ entsprechen ${ }^{1}$ ). In den Punkten $P_{g o}$ und $P_{g_{\infty}}$ berührt daher der gesuchte Grundkreis

1) $\mathrm{Da}$ sich für den Halbmesser $\mathrm{R}\left(\mathrm{R}_{25}=0\right)$ in praktischen Fällen ein sehr grofer Wert. ergibt, können die Punkte $P_{g o}$ und $P_{g o o}$ in der Weise ermittelt werden, daf auf der u'-Achse eine dem Halbmesser $R_{\left(R_{2 s}=o\right)}$ proportionale Strecke und senkrecht dazu die im gleichen Verhältnis verkleinerten Halbmesser $R_{h o}$ und $R_{h \infty}$ der Hilfskreise $K_{h o}$ und $K_{h \infty}$ aufgetragen werden. Die Verbindungsgeraden der so erhaltenen Punkte mit $O^{\prime}$ schneiden die beiden Hilfskreise in den gesuchten Punkten $P_{g \circ}$ und $P_{g o \infty}$. 
die Hilfskreise; sein Mittelpunkt $\mathrm{M}_{\mathrm{g}}$ ist durch den Schnitt der Mittelsenkrechten der Strecke $\overline{\mathrm{P}}_{\mathrm{g}_{0}} \mathrm{P}_{\mathrm{g} \infty}$ mit der Verbindungsgeraden $\mathrm{P}_{\mathrm{g} \infty} \mathrm{M}_{\mathrm{h} \infty}$ bestimmt.

Um den der Schlüpfung $\mathrm{s}=\mathrm{I}$ entsprechenden Punkt $\mathrm{P}_{\mathrm{g} \text { St }}$ des Grundkreises zu ermitteln, tragen wir in Analogic zur Abb. 8 auf dem Mittelpunktsstrahle $\mathrm{P}_{\mathbf{g}} \mathrm{M}_{\mathbf{g}}$ die Strecke

und senkrecht dazu

$$
\overline{\mathrm{P}_{\mathrm{go}} \mathrm{f}^{\prime \prime}}=\mathrm{x}_{\mathrm{II}}^{*}+\dot{\mathrm{x}}_{3}
$$

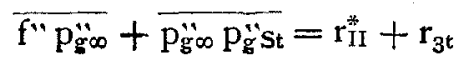

auf. Die Verbindungsgerade $\mathrm{P}_{\mathrm{g}} \mathrm{P}_{g}$ 'st schneidet den Grundkreis im Punkte $\mathrm{P}_{\mathrm{g}} \mathrm{St}$. Die Punkte $P_{g o}, p_{g \infty o}$ und $P_{g \infty}$ müssen bei richtiger Konstruktion auf einer Geraden liegen.

Bei der Besprechung der $\left(\mathfrak{z}_{1}^{*}+\beta_{2 s}\right)$-Kurve im Abschnitte 4, Seite I I, wurde bemerkt, daß die sich beiderseits ihres Synchronismuspunktes $p_{0}$ bis zum Doppelpunkte $p_{d}$ erstreckende Schleife derselben von dem in Abb. 7 gezeichneten Kreise $k_{n}$ mit dem Mittelpunkte $m_{n}$ eingeschlossen wird, welcher durch Verschiebung des Kreises $k_{g}$ um den Betrag $\frac{r_{2}}{\beta}$ parallel zur v'-Achse erhalten wurde. Die parallel zur $v^{\prime}$-Achse gezogenen Tangenten des Kreises $k_{\mathbf{q}}$ berühren den Kreis $k_{\mathfrak{n}}$ in den Punkten $p_{0}$ und $b_{n}$. Es wird demnach auch die entsprechende Schleife der Kaskadenkurve von einem Kreise $K_{n}\left(P_{o}\right)$ eingeschlossen werden, welcher aus $k_{n}$ durch Inversion in bezug auf den Pol $\mathrm{O}^{\prime}$ abgeleitet werden kann. Es ist klar, daß der Kreis $\mathrm{K}_{\mathrm{n}}\left(\mathrm{P}_{\mathrm{o}}\right)$ die in Abb. 12 gezeichneten Hilfskreise $\mathrm{K}_{\mathrm{ho}}$ und $\mathrm{K}_{\mathrm{h} \infty}$ berühren muß.

Für den $\mathrm{Kreis} \mathrm{K}_{\mathrm{n}}\left(\mathrm{P}_{0}\right)$ ergibt sich nach dieser Uberlegung folgende Konstruktion. Wir ermitteln zunächst nach dem im Abschnitte 6, Seite 17 , angegebenen Verfahren aus dem Punkte $P_{g_{0}}$ des Grundkreises den Synchronismuspunkt $P_{o}$ des Kaskadendiagramms (in welchem der $\mathrm{Kreis} \mathrm{K}_{\mathrm{n}}\left(\mathrm{P}_{\mathrm{o}}\right)$ den Hilfskreis $\mathrm{K}_{\mathrm{ho}}$ berührt), indem wir im Abstand

$$
\overline{\mathrm{O}^{\prime} \mathrm{u}_{\mathrm{o}}}=\frac{\mathrm{I}}{2 \mathrm{C}_{1}^{2}} \cdot \frac{\beta}{\mathrm{r}_{2}}
$$

von $O^{\prime}$ eine Senkrechte zur u'-Achse ziehen, sie mit der Verbindungsgeraden $O^{\prime} \mathrm{P}_{\mathrm{g} o}$ zum Schnitte bringen und von dem Schnittpunkte $\pi_{\mathrm{s} o}$ den Halbmesser $R_{\mathrm{ho}_{\mathrm{o}}}=\bar{\pi}_{\mathrm{go}} \bar{\pi}_{\mathrm{o}}$ des Hilfskreises $K_{h o}$ auftragen; die Verbindungsgerade $O^{\prime} \pi_{0}$ schneidet den Hilfskreis $K_{h o}$ im Punkte $P_{o}$. In ganz ähnlicher Weise läßt sich auch der Berührungspunkt $B_{n}$ des gesuchten Kreises mit dem Hilfskreise $K_{h \infty}$ finden, wenn wir von dem Schnittpunkte der Verbindungsgeraden $\mathrm{O}^{\prime} \mathrm{P}_{\mathrm{g} \infty}$ mit der früher gezogenen Senkrechten den Halbmesser $R_{h \infty}$ auftragen; die Verbindungsgerade des so erhaltenen Punktes mit $O^{\prime}$ schneidet den Hilfskreis im Punkte $B_{n}$. Der Mittelpunkt $\mathrm{M}_{n}\left(\mathrm{P}_{\mathrm{o}}\right)$ des Kreises $K_{n}\left(P_{o}\right)$ ergibt sich schließlich im Schnitte der Mittelsenkrechten der Strecke $\overline{P_{o} B_{n}}$ mit der Verbindungsgeraden $B_{n} M_{h \infty}$. Der genannte Kreis schließt die sich beiderseits des Synchronismuspunktes $\mathrm{P}_{o}$ bis zum Doppelpunkt $\mathrm{P}_{\mathrm{d}}$ erstreckende Schleife der Kaskadenkurve ein.

Für eine rasche Orientierung über den Verlauf der Kaskadenkurve empfiehlt sich die Ermittlung des Doppelpunktes $P_{d}$, welcher aus den durch die. Schlüpfungs: werte $\pm s_{d}(83)$ bestimmten Punkten des Grundkreises abgeleitet werden kann. Der den Schlüpfungswerten $\pm \infty$ entsprechende Punkt $\mathrm{P}_{\infty}$ der Kaskadenkurve fällt mit dem Punkte $\mathrm{P}_{\mathrm{g} 8}$ des Grundkreises zusammen. Vom Punkte $\mathrm{P}_{\infty}$ an entfernt sich die Kurve vom Hilfskreis $K_{h \infty}$, passiert den Doppelpunkt $P_{d}$ und nähert sich hierauf dem Kreise $K_{n}\left(P_{0}\right)$, bis zum Synchronismuspunkte $P_{o}$. Im weiteren Verlaufe entfernt sich die Kurve wieder von dem Kreise $K_{n}\left(P_{o}\right)$, passiert ein zweites Mal den Doppelpunkt $P_{d}$ und nähert sich schließlich dem Hilfskreise $K_{h \infty}$ bis zum Punkte 
$\mathrm{O}^{\prime} \equiv \mathrm{P}_{-} \frac{\beta}{\alpha}$. Von hier an läßt sich die Kaskadenkurve mit praktisch genügender Annäherung durch den Hilfskreis $\mathrm{K}_{\mathrm{h} \infty}$ ersetzen, wenn der tertiäre Werkwiderstand nicht zu groß ist.

\section{Darstellung der primären elektrischen Leistung, der Drehmomente} und mechanischen Leistungen im Stromdiagramm der Kaskadenschaltung (alles pro Phase gerechnet).

In $\mathrm{Abb}$. Io ist das Kaskadendiagramm mit dem Grundkreise $\mathrm{K}_{\mathrm{g}}$ nochmals gezeichnet, welches im folgenden als Strom diagramm der Kaskadenschaltung angesehen werden soll.

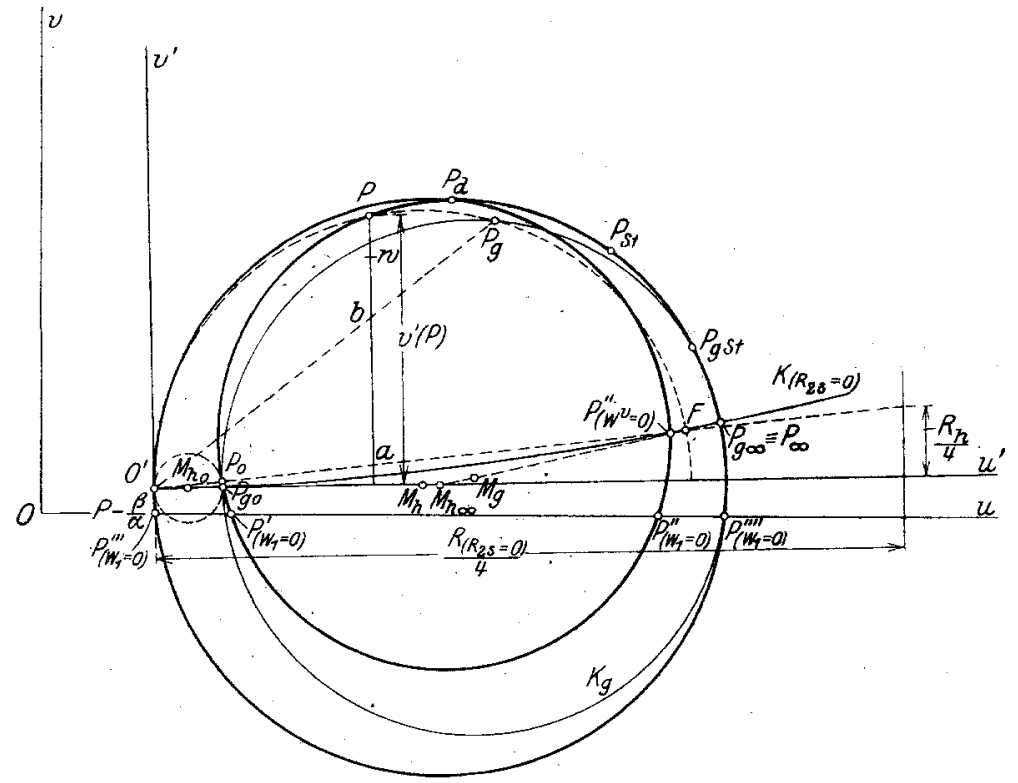

Abb. ro.

Die primäre elektrische Leistung

$$
\mathrm{W}_{1}=\mathrm{P}_{1} \mathrm{I}_{1} \cos \varphi_{1}=\mathrm{P}_{1} \mathrm{v}(\mathrm{P})_{\text {strom }}
$$

wird in diesem Diagramm bei konstanter primärer Klemmenspannung $P_{1}$ durch die auf ( $u$ v) bezogenen Ordinaten $v(P)_{\text {Strom }}$ der Diagrammpunkte im Maßstabe $\frac{W_{1}}{P_{1}}$ gemessen. In $\mathrm{Abb}$. I I b sind die der Abb. Io entnommenen Ordinaten $\mathrm{v}(\mathrm{P})$ in $\mathrm{Ab}-$ hängigkeit von der Schlüpfung $s$ eingetragen.

Um die analytischen Ausdrücke für die Komponenten des Primärstromes und die primäre elektrische Leistung zu erhalten, führen wir in 49a) den mit Hilfe des vektoriellen Verhältnisses zwischen Primär- und Sekundärstrom

gebildeten Scheinwiderstand

$$
\widetilde{E}_{2, \mathrm{~s}}=\frac{\widetilde{\Im}_{1}}{\widetilde{\Im}_{2}}=\frac{z_{\mathrm{a}}+\mathscr{\bigotimes}_{\mathrm{as}}}{z_{\mathrm{a}}}
$$

$$
Z_{2 s}^{*}=\frac{Z_{2 s}}{\mathfrak{C}_{2 s}}=R_{2 s}^{*}+j X_{2 s}^{*}
$$

ein und erhalten

$$
\dddot{\mho}_{1}=\frac{\mathfrak{P}_{1}}{\mathfrak{z}_{1}+\mathfrak{Z}_{2 \mathrm{~s}}^{*}}=\frac{\mathfrak{P}_{1}}{\left(\mathrm{r}_{1}+\mathrm{R}_{2 \mathrm{~s}}^{*}\right)+\mathrm{j}\left(\mathrm{x}_{1}+\mathrm{X}_{2 \mathrm{~s}}^{*}\right)}
$$




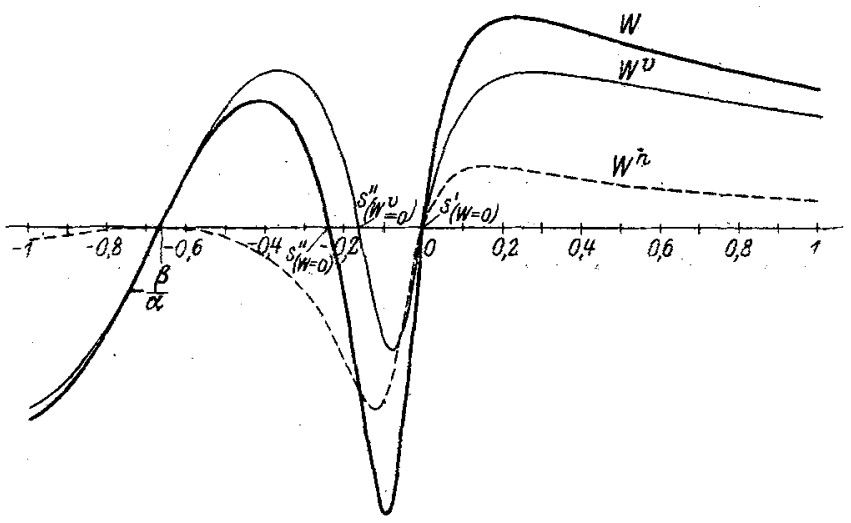

Abb. I a.

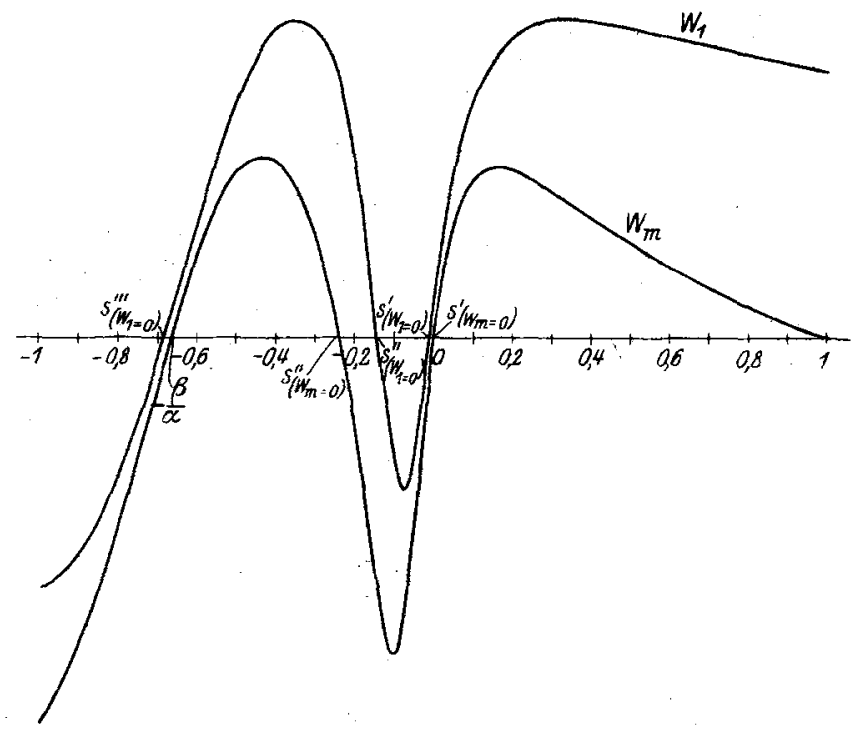

Abb. II b.

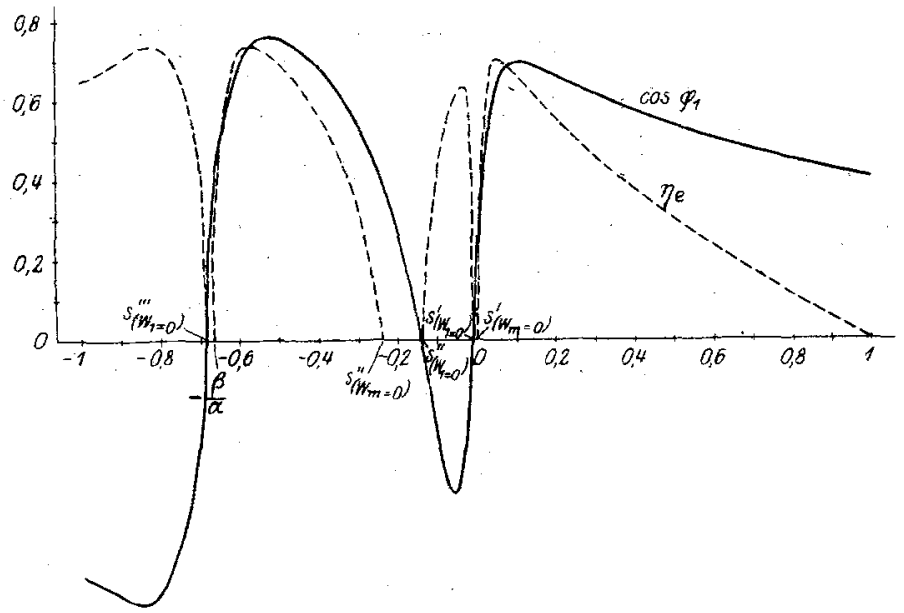

Abb. II c. 
Die Komponenten von $\bigcap_{2 s}^{*}$ sind

wobei

$$
\begin{aligned}
& \mathrm{R}_{2 \mathrm{~s}}^{*}=\frac{\mathrm{I}}{\mathrm{C}_{2 \mathrm{~s}}^{2}} \cdot\left[\mathrm{r}_{\mathrm{a}}\left(\frac{\mathrm{Z}_{2 \mathrm{~s}}}{\mathrm{Z}_{\mathrm{a}}}\right)^{2}+\mathrm{R}_{2 \mathrm{~s}}\right], \\
& \mathrm{X}_{2 \mathrm{~s}}^{*}=\frac{\mathrm{I}}{\mathrm{C}_{2 \mathrm{~s}}^{2}} \cdot\left[\mathrm{X}_{\mathrm{a}}\left(\frac{\mathrm{Z}_{2 \mathrm{~s}}}{Z_{\mathrm{a}}}\right)^{2}+\mathrm{X}_{2 \mathrm{~s}}\right],
\end{aligned}
$$

$$
C_{2 s}^{2}=\frac{\left(r_{a}+R_{a s}\right)^{2}+\left(x_{a}+X_{a s}\right)^{2}}{z_{a}^{2}}
$$

das Quadrat des Betrages von $\mathfrak{C}_{2 \mathrm{~s}}$ darstellt.

Aus 97) ergeben sich die auf ( $u$ v) bezogenen Komponenten des Primärstromes, welche mit den Koordinaten der Punkte P des Stromdiagramms identisch sind, zu

$$
\begin{aligned}
& \mathrm{I}_{1} \cos \varphi_{1}=\mathrm{v}(\mathrm{P})_{\text {Strom }}=\mathrm{P}_{1} \cdot \frac{\mathrm{r}_{1}+\mathrm{R}_{2 \mathrm{~s}}^{*}}{\left(\mathrm{r}_{1}+\mathrm{R}_{2 \mathrm{~s}}^{*}\right)^{2}+\left(\mathrm{x}_{1}+\mathrm{X}_{2 \mathrm{~s}}^{*}\right)^{2}} \\
& \mathrm{I}_{1} \sin \varphi_{1}=\mathrm{u}(\mathrm{P})_{\text {Strom }}=\mathrm{P}_{1} \cdot \frac{\mathrm{x}_{1}+\mathrm{X}_{2 \mathrm{~s}}^{*}}{\left(\mathrm{r}_{1}+\mathrm{R}_{2 \mathrm{~s}}^{*}\right)^{2}+\left(\mathrm{x}_{1}+\mathrm{X}_{2 \mathrm{~s}}^{*}\right)^{2}}
\end{aligned}
$$

Da

ist auch

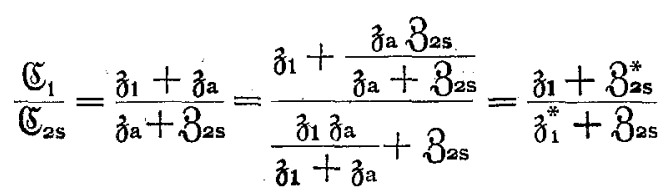

$$
\mathfrak{J}_{1}=\mathfrak{P}_{1} \cdot \frac{\mathfrak{S}_{2 \mathrm{~s}}}{\mathfrak{S}_{1}} \cdot \frac{1}{\mathfrak{\jmath}_{1}^{*}+\beta_{\mathrm{as}}}
$$

Ferner gilt die Identität

$$
\left(r_{1}+R_{2 s}^{*}\right)^{2}+\left(x_{1}+X_{2 s}^{*}\right)^{2}=\frac{C_{1}^{2}}{C_{2 s}^{2}} \cdot\left[\left(r_{1}^{*}+R_{2 s}\right)^{2}+\left(x_{1}^{*}+X_{2 s}\right)^{2}\right]
$$

wenn wir den Nenner der Gleichungen 100) durch den letzteren Ausdruck ersetzen und in den Zählern für $R_{a s}^{*}$ bzw. $X_{2 e}^{*}$ die Ausdrücke 98) einführen, wird

$$
\begin{aligned}
& I_{1} \cos \varphi_{1}=v(P)_{\text {Strom }}=\frac{P_{1}}{C_{1}^{2}} \cdot \frac{r_{1} C_{2 s}^{2}+r_{a}\left(\frac{Z_{2 s}}{z_{a}}\right)^{2}+R_{2 s}}{\left(r_{1}^{*}+R_{2 s}\right)^{2}+\left(x_{1}^{*}+X_{2 s}\right)^{2}}, \\
& I_{1} \sin \varphi_{1}=u(P)_{\text {Strom }}=\frac{P_{1}}{C_{1}^{2}} \cdot \frac{x_{1} C_{2 s}^{2}+x_{a}\left(\frac{Z_{2 s}}{z_{a}}\right)^{2}+X_{2 s}}{\left(r_{1}^{*}+R_{2 s}\right)^{2}+\left(x_{1}^{*}+X_{2 s}\right)^{2}} .
\end{aligned}
$$

Durch Multiplikation der ersten Gleichung 100 a) mit dem Betrage $P_{1}$ der primären Klemmenspannung ergibt sich schließlich die primäre elektrische Leistung

$$
\mathrm{W}_{1}=\mathrm{P}_{1} \mathrm{I}_{1} \cos \varphi_{1}=\frac{\mathrm{P}_{1}^{2}}{\mathrm{C}_{1}^{2}} \cdot \frac{\mathrm{r}_{1} \mathrm{C}_{2 \mathrm{~s}}^{2} \perp \mathrm{r}_{2}\left(\frac{\mathrm{Z}_{\mathrm{as}}}{\mathrm{z}_{\mathrm{a}}}\right)^{2}+\mathrm{R}_{2 \mathrm{~s}}}{\left(\mathrm{r}_{1}^{*}+\mathrm{R}_{2 \mathrm{~s}}\right)^{2}+\left(\mathrm{x}_{1}^{*}+\mathrm{X}_{2 \mathrm{~s}}\right)^{2}}
$$
stromes

Wenn wir andererseits die erste Gleichung rooa) durch den Betrag des Primär-

$$
\mathrm{I}_{1}=\mathrm{P}_{1} \cdot \frac{\mathrm{C}_{2 \mathrm{~s}}^{*}}{\mathrm{C}_{1}} \cdot \frac{\mathrm{I}}{\sqrt{\left(\mathrm{r}_{1}^{*}+\mathrm{R}_{2 \mathrm{~s}}\right)^{2}+\left(\mathrm{x}_{1}^{*}+\mathrm{X}_{2 \mathrm{~s}}\right)^{2}}} \text { (aus 97a) abzulesen) }
$$

dividieren, erhalten wir den primären Leistungsfaktor 


$$
\cos \varphi_{1}=\frac{\mathrm{I}}{\mathrm{C}_{1} \mathrm{C}_{2 \mathrm{~s}}} \cdot \frac{\mathrm{r}_{1} \mathrm{C}_{2 \mathrm{~s}}^{2}+\mathrm{r}_{\mathrm{a}}\left(\frac{\mathrm{Z}_{2 \mathrm{~s}}}{z_{2}}\right)^{2}+\mathrm{R}_{2 \mathrm{~s}}}{\sqrt{\left(\mathrm{r}_{1}^{*}+\mathrm{R}_{2 \mathrm{~s}}\right)^{2}+\left(\mathrm{x}_{1}^{*}+\mathrm{X}_{2 \mathrm{~s}}\right)^{2}}} .
$$

Die dem Kaskadendiagramm Abb. Io entsprechende Abhängigkeit dieses Faktors von der Schlüpfung $s$ ist aus $A b b$. I I c zu ersehen.

Nach dem Ersatzstromkreis Abb. 5 a läßt sich die primäre elektrische Leistung in die Leistungssumme

$$
W_{1}=I_{1}^{2} r_{1}+I_{a}^{2} r_{a}+I_{2}^{2} R_{2 s}
$$

zerlegen; $I_{1}^{2} r_{1}$ sind die Kupferverluste in der Primärwicklung und $I_{a}^{2} r_{a}$ die Eisenverluste im Ständer der vorderen Maschine. Die nach Abzug dieser Verluste von $\mathrm{W}_{1}$ übrig bleibende Leistung

$$
W_{d}^{v}=W_{1}-I_{1}^{2} r_{1}-I_{2}^{2} r_{a}=I_{2}^{2} R_{2 s}
$$

wird in den Motorarbeitsgebieten der Kaskadenschaltung auf das mit $N_{1}$ minutlichen Umdrehungen rotierende Drehfeld der vorderen Maschine übertragen; wir wollen sie die "Drehfeldleistung der vorderen Maschine" nennen. Diese Leistung wird im Stromdiagramm nach 54) durch die Abschnitte

$$
\overline{\mathrm{Pa}}_{\text {Strom }}=\frac{\mathrm{I}_{2}^{2} \mathrm{R}_{2 \mathrm{~s}}}{\mathrm{P}_{1}}=\frac{\mathrm{W}_{\mathrm{d}}^{\mathrm{v}}}{\mathrm{P}_{1}}
$$

der auf ( $u^{\prime} v$ ') bezogenen Ordinaten $v^{\prime}(P)_{\text {Strom }}$ der Diagrammpunkte in demselben Maßstabe dargestellt, wie die primäre elektrische Leistung $W_{1}$ durch die Ordinaten $\mathrm{v}(\mathrm{P})_{\text {Strom }}$.

Die Punkte a liegen nach Abb. 7 im Schnitte der Verbindungsgeraden $\mathrm{O}^{\prime} \mathrm{f}_{3}$ mit den Ordinaten $v^{\prime}(\mathrm{P})$; die Punkte $\mathrm{f}_{\mathbf{3}}$ sind die Fußpunkte der Ordinaten $v^{\prime}\left(\mathrm{p}_{\mathrm{g}}\right)$ des. Kreises $k_{\mathrm{g}}$ auf der im Abstande $r_{1}^{*}$ parallel zur $u^{\prime}$-Achse verlaufenden $u_{3}$-Achse. Um die Punkte $a$ in dem direkt auf dem Grundkreise $K_{g}$ aufgebauten Kaskadendiagramm zu ermitteln, denken wir uns die Parallelen zur v'-Achse (auf welchen die Ordinaten $v^{\prime}\left(\mathrm{p}_{\mathrm{g}}\right)$ liegen) und die $\mathrm{u}_{3}$-Achse in bezug auf den Pol $\mathrm{O}^{\prime}$ mit Berücksichtigung des Reduktionsverhältnisses $\frac{\mathbf{I}}{\mathrm{C}_{1}^{2}}$ inversiert. Die genannten Parallelen gehen dabei in die Hilfskreise über, die $u_{3}$-Achse inversiert sich in den $\mathrm{Kreis} \mathrm{K}_{\left(\mathrm{R}_{25}\right.}=0$ ) mit dem Durchmesser (im Strommaßstabe)

$$
2 R_{\left(R_{2 s}=0\right)}=\frac{P_{1}}{C_{1}^{2}} \cdot \frac{I}{r_{1}^{*}},
$$

welcher durch den $\mathrm{Pol} \mathrm{O}^{\prime}$ geht und dessen Mittelpunkt auf der 'v'-Achse liegt. Auf diesem Kreise liegen nach früherem (S. 20) auch die Punkte $\mathrm{P}_{g_{0}}$ und $\mathrm{P}_{\mathrm{g}_{\infty}}$ des Grundkreises.

Die Schnittpunkte $F$ des Kreises $K_{\left(R_{2 s}\right.}=0$, mit den durch die Punkte $P$ des Kaskadendiagramms gezogenen Hilfskreisen entsprechen inversionsgemäß den Fußpunkten $\mathrm{f}_{3}$ auf der $\mathrm{u}_{3}$-Achse; die Verbindungsgeraden $\mathrm{O}^{\prime} \mathrm{F}$ schneiden daher die Ordinaten $v^{\prime}(P)$ in den gesuchten Punkten a, wobei zusammengehörige Punkte $F$ und $P$ auf einem Hilfskreise liegen. - Die oberhalb (unterhalb) der Verbindungsgeraden $\mathrm{O}^{\prime} \mathrm{F}$ liegenden Ordinatenabschnitte $\overline{\mathrm{Pa}}$ sind positiv (negativ) zu rechnen.

Wenn wir die Drehfeldleistung $W_{d}^{v}$ der vorderen Maschine, welche der Umdrehungszahl $\mathrm{N}_{1}$ ihres Drehfeldes entspricht, durch Multiplikation mit dem. Verhältnis $\frac{\mathrm{n}_{10}}{\mathrm{~N}_{1}}=\alpha(6 \mathrm{a})$ auf die synchrone Umdrehungszahl $\mathrm{n}_{10}$ reduzieren, erhalten wir das Drehmoment der vorderen Maschine in synchronen Watt

$$
\mathrm{W}^{\mathrm{v}}=\alpha \mathrm{W}_{\mathrm{d}}^{\mathrm{v}}=\alpha \mathrm{I}_{\mathrm{a}}^{2} \mathrm{R}_{\mathrm{as}}
$$

die mèhanische Leistung der vorderen Maschine ist dann 


$$
\mathrm{W}_{\mathrm{m}}^{\mathrm{v}}=\frac{\mathrm{n}_{1}}{\mathrm{n}_{10}} \cdot \mathrm{W}^{\mathrm{v}}=(\mathrm{I}-\mathrm{s}) \alpha \mathrm{I}_{2}^{2} \mathrm{R}_{\mathbf{2 s}}
$$

Die Leistungen $W^{v}$ und $W_{m}^{v}$ lassen sich auf die im Strommaßstabe gemessenen Ordinatenabschnitte $\overline{\text { Pa }}_{\text {Strom }}$ beziehen, und zwar ist mit Rücksicht auf IO4)

und

$$
\alpha \cdot \overline{\mathrm{Pa}}_{\mathrm{Strom}}=\frac{\mathrm{W}^{\mathrm{v}}}{\mathrm{P}_{1}}
$$

$$
(\mathrm{I}-\mathrm{s}) \alpha \cdot \overline{\mathrm{Pa}}_{\text {Strom }}=\frac{\mathrm{W}_{\mathrm{m}}^{\mathrm{v}}}{\mathrm{P}_{1}}
$$

In $\mathrm{Abb}$. I I a sind die aus den Ordinatenabschnitten $\overline{\mathrm{Pa}}$ der Abb. 1o ermittelten Drehmomente der vorderen Maschine in Abhängigkeit von der Schlüpfung $s$ eingetragen.

Die Differenz zwischen der Drehfeldleistung $W_{d}^{v}$ und der mechanischen Leistung der vorderen Maschine $W_{m}^{\mathbf{v}}$

$$
\mathrm{W}_{2}=[\mathrm{I}-(\mathrm{I}-\mathrm{S}) \alpha] \mathrm{I}_{\mathrm{z}}^{2} \mathrm{R}_{2 \mathrm{~s}}=(\alpha \mathrm{s}+\beta) \mathrm{I}_{2}^{2} \mathrm{R}_{2 \mathrm{~s}}
$$

wird in den Motorarbeitsgebieten dem Sekundärkreise der Kaskadenschaltung in Form elektrischer Leistung zugeführt.

Die dem zusammengefaßten Scheinwiderstande

$$
8_{2 s}=R_{2 s}+j X_{2}=\left(\frac{r_{2}}{\alpha s+\beta}+r_{3 s}^{*}\right)+j\left(x_{2}+x_{3 s}^{*}\right)
$$

entsprechende Leistung $I_{2}^{2} R_{2 s}$ läßt sich in zwei Summanden zerlegen:

$$
\mathrm{I}_{2}^{2} \mathrm{R}_{2 \mathrm{~s}}=\mathrm{I}_{2}^{2} \cdot \frac{\mathrm{r}_{2}}{\alpha \mathrm{s}+\beta}+\mathrm{I}_{2}^{2} \mathrm{r}_{3 \mathrm{~s}}^{*}
$$

die Multiplikation dieser Gleichung mit $\alpha \mathrm{s}+\beta$ ergibt

$$
(\alpha \mathrm{s}+\beta) \mathrm{I}_{2}^{2} \mathrm{R}_{2 \mathrm{~s}}=\mathrm{I}_{2}^{2} \mathrm{r}_{2}+(\alpha \mathrm{s}+\beta) \mathrm{I}_{2}^{2} \mathrm{r}_{3 \mathrm{~s}}^{*} \text {. }
$$

$(\alpha s+\beta) I_{2}^{2} R_{2 s}$ ist nach IIO). die dem Sekundärkreise der Kaskadenschaltung zugeführte elektrische Leistung $\mathrm{W}_{2}, \mathrm{I}_{2}^{2} \mathrm{r}_{2}$ sind die Kupferverluste in den hintereinander geschalteten Wicklungen der beiden Maschinen. Die nach Abzug dieser Verluste von $\mathrm{W}_{2}$ übrig bleibende Leistung

$$
\mathrm{W}_{\mathrm{d}}^{\mathrm{h}}=(\alpha \mathrm{s}+\beta) \mathrm{I}_{2}^{2} \mathrm{r}_{3 \mathrm{~s}}^{*} \text {, }
$$

welche auf das mit $\mathrm{N}_{2}$ minutlichen Umdrehungen rotierende Drehfeld der hinteren Maschine übertragen wird, soll als „Drehfeldleistung der hinteren Maschine“ bezeichnet werden.

Wenn wir die Leistung $W_{d}^{\mathrm{h}}$ durch Multiplikation mit dem Verhältnis $\frac{\mathrm{n}_{20}}{\mathrm{~N}_{2}}=\frac{\beta}{\alpha \mathrm{s}+\beta}(7 \mathrm{~b})$ auf die synchrone. Umdrehungszahl $\mathrm{n}_{20}$ reduzieren, erhalten wir das Drehmoment der hinteren Maschine in synchronen Watt

$$
\mathrm{W}^{\mathrm{h}}=\beta \mathrm{I}_{2}^{2} \mathrm{r}_{3 \mathrm{~s}}^{*}
$$

und daraus ihre mechanische Leistung

$$
\mathrm{W}_{\mathrm{m}}^{\mathrm{h}}=(\mathrm{I}-\mathrm{s}) \mathrm{W}^{\mathrm{h}}=(\mathrm{I}-\mathrm{s}) \beta \mathrm{I}_{2}^{2} \mathrm{r}_{3 \mathrm{~s}}^{*} \text {. }
$$

Die Leistungen $I_{2}^{2} r_{3}^{*}$ werden nach 55) durch die Abschnitte

$$
\overline{\mathrm{ab}}_{\mathrm{Strom}}=\frac{\mathrm{I}_{2}^{2} \overline{\mathrm{r}}_{3 \mathrm{~s}}^{*}}{\overline{\mathrm{P}}_{1}}
$$

der auf ( $\left.u^{\prime} v^{\prime}\right)$ bezogenen Ordinaten $v^{\prime}(P)$ der Punkte $P$ des Kaskadendiagramms in demselben Maßstabe dargestellt, wie die Drehfeldleistungen der vorderen Maschine $\mathrm{W}_{\mathrm{d}}^{\mathrm{v}}$ durch die Abschnitte $\overline{\mathrm{Pa}}_{\text {Strom. }}$ Die Punkte b liegen nach Abb. 7 im Schnitte der Verbindungsgeraden $\mathrm{O}^{\prime} \mathrm{P}_{\mathbf{g}}$. mit den Ordinaten $v^{\prime}(\mathrm{P})$. 
Bei der Ermittlung der Abschnitte $\bar{a}_{\text {Strom }}$ ist auf die richtige Zugehörigkeit ihrer Begrenzungspunkte zu achten; diese Abschnitte sind für positive (negative) Schlüpfungswerte $s$ positiv (negativ) zu rechnen.

Die Leistungen $W^{h}$ und $W_{m}^{h}$ lassen sich aus den Ordinatenabschnitten $\bar{a}_{\text {strom }}$ mit Hilfe der Beziehungen

und

$$
\beta \cdot \overline{\mathrm{ab}}_{\mathrm{Strom}}=\frac{\mathrm{W}^{\mathrm{h}}}{\mathrm{P}_{1}}
$$

$$
(\mathrm{I}-\mathrm{s}) \beta \cdot \overline{\mathrm{ab}}_{\mathrm{Strom}}=\frac{\mathrm{W}_{\mathrm{m}}^{\mathrm{h}}}{\mathrm{P}_{\mathrm{i}}}
$$

in demselben Maßstab ermitteln, wie die entsprechenden Größen der vorderen Maschine $\mathrm{W}^{\mathrm{v}}$ und $\mathrm{W}_{\mathrm{m}}^{\mathrm{v}}$ aus den Abschnitten $\overline{\mathrm{Pa}}_{\text {Strom. }}$. In $\mathrm{Abb}$. I I a sind die der $\mathrm{Abb}$. Io entsprechenden Leistungen $W^{h}$ im gleichen Maßstabe wie $W^{v}$ dargestellt.

Das Gesamtdrehmoment in synchronen Watt der beiden in Kaskade geschalteten Maschinen ergibt sich durch Summierung der Drehmomente $W^{v}$ und $\mathrm{W}^{\mathrm{h}} \mathrm{zu}$

wenn wir hierin

$$
\mathrm{W}=\mathrm{W}^{\mathbf{v}}+\mathrm{W}^{\mathrm{h}}=\mathrm{I}_{2}^{2}\left(\alpha \mathrm{R}_{\mathrm{as}}+\beta \mathrm{r}_{3 \mathrm{~s}}^{*}\right)
$$

$$
\mathrm{R}_{2 \mathrm{~s}}=\frac{\mathrm{r}_{2}}{\alpha \mathrm{s}+\beta}+\mathrm{r}_{3 \mathrm{~s}}^{*}
$$

setzen, wird $\mathrm{W}=\mathrm{I}_{2}^{2}\left[\alpha \cdot \frac{\mathrm{r}_{2}}{\alpha \mathrm{s}+\beta}+(\alpha \mathrm{s}+\beta) \mathrm{r}_{3 \mathrm{~s}}^{*}\right]=\mathrm{I}_{2}^{2}\left[\alpha \cdot \frac{\mathrm{r}_{2}}{\alpha \mathrm{s}+\beta}+\mathrm{r}_{3 \mathrm{~s}}^{*}\right]$.

Das Gesamtdrehmoment W läßt sich im Kaskadendiagramm in verhältnismäßig einfacher Weise darstellen. Nach 56) sind die Abschnitte

$$
\overline{\mathrm{Pb}}_{\text {Strom }}=\frac{\mathrm{I}_{2}^{2} \cdot \frac{\mathrm{r}_{2}}{\alpha \mathrm{s}+\beta}}{\mathrm{P}_{1}}
$$

der Ordinaten $v^{\prime}(\mathrm{P})$ den Leistungen $\mathrm{I}_{2}^{2} \cdot \frac{\mathrm{r}_{2}}{\alpha \mathrm{s}+\beta}$ proportional; im gleichen Maßstabe messen die Abschnitte $\overline{\mathrm{ab}}_{\text {Strom }}$ dieser Ordinaten die Leistungen $\mathrm{I}_{\mathbf{2}}^{2} \mathrm{r}_{3 \mathrm{~s}}^{*}$. Die Abschnitte $\overrightarrow{\mathrm{Pb}}$ sind dabei positiv (negativ) zu rechnen, wenn $(\alpha \mathrm{s}+\beta)$ positiv (negativ) ist; die Abschnitte $\overline{\mathrm{ab}}$ sind dagegen nach früherem positiv (negativ) für positive (negative) Schlüpfungswerte. Wenn wir demnach in $\mathrm{Abb}$. Io die Abschnitte $\overline{\mathrm{ab}}$ mit den dem Verhältnis $\alpha$ proportionalen Teilen

$$
\overline{\mathrm{bw}}=\alpha \cdot \overline{\mathrm{Pb}}
$$

der Abschnitte $\overline{\mathrm{Pb}}$ unter Berücksichtigung ihrer Vorzeichen zusammensetzen, so sind die resultierenden Strecken $\overline{\mathrm{a} w}$ den Leistungen

$$
I_{2}^{2}\left(\alpha \cdot \frac{r_{2}}{\alpha s+\beta}+r_{3 s}^{*}\right)
$$

und damit nach IIIa) dem Gesamtdrehmomente $W$ der Kaskadenschaltung proportional. Für $\alpha=\beta$ sind die Abschnitte $\overline{\mathrm{Pb}}$ einfach zu halbieren.

Im Stromdiagramm messen die Strecken

$$
\overline{\mathrm{a}}_{\text {Strom }}=\frac{\mathrm{I}_{2}^{2}\left(\alpha \cdot \frac{\mathrm{r}_{2}}{\alpha \mathrm{s}+\beta}+\mathrm{r}_{3 \mathrm{~s}}^{*}\right)}{\mathrm{P}_{1}}=\frac{\mathrm{W}}{\mathrm{P}_{1}}
$$

das Gesamtdrehmoment in synchronen Watt $W$ in dem uns schon bekannten Maßstabe. 
Aus dem Gesamtdrehmomente $W$ ergibt sich die gesamte mechanische Leistung

$$
\mathrm{W}_{\mathrm{m}}=(\mathrm{r}-\mathrm{s}) \mathrm{W}=(\mathrm{I}-\mathrm{s}) \mathrm{I}_{2}^{2}\left(\alpha \cdot \frac{\mathrm{r}_{2}}{\alpha \mathrm{s}+\beta}+\mathrm{r}_{3 \mathrm{~s}}^{*}\right)
$$

Wenn in 118 a) und 122 ) für $I_{2}^{2}$ der Wert aus 53) eingesetzt wird, resultieren die folgenden Ausdrücke für das Gesamtdrehmoment in synchronen Watt

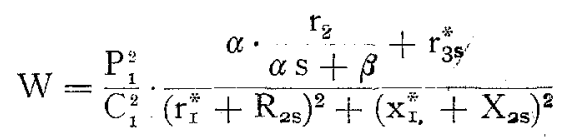

und die gesamte mechanische Leistung der beiden Maschinen

$$
\mathrm{W}_{\mathrm{m}}=\frac{\mathrm{P}_{1}^{2}}{\mathrm{C}_{1}^{*}} \cdot \frac{(\mathrm{I}-\mathrm{s})\left(\alpha \cdot \frac{\mathrm{r}_{2}}{\alpha \mathrm{s}+\beta}+\mathrm{r}_{3 \mathrm{~s}}^{*}\right)}{\left(\mathrm{r}_{\mathrm{I}}^{*}+\mathrm{R}_{2 \mathrm{~s}}\right)^{2}+\left(\mathrm{x}_{\mathrm{I}}^{*}+\frac{\left.\mathrm{X}_{2 \mathrm{~s}}\right)^{2}}{\mathrm{z}^{2}} .\right.}
$$

In den Motorarbeitsgebieten der Kaskadenschaltung sind von $\mathrm{W}_{\mathrm{m}}$ die Reibungsverluste abzuziehen, um die nutzbare mechanische Leistung zu erhalten; in den Generatorarbeitsgebieten sind die Reibungsverluste zu addieren.

Aus Abb. I I a ist der dem Kaskadendiagramm Abb. Io entsprechende Verlauf des Gesamtdrehmoments W, aus Abb. I I b der Verlauf der gesamten mechanischen Leistung zu ersehen.

Durch Division von 122a) und 94a) ergibt sich schließlich der den berücksichtigten Verlusten entsprechende elektrische Wirk ungsgrad der Kaskadenschaltung in den Motorarbeitsgebieten

$$
\eta_{\mathrm{e}}=\frac{\mathrm{W}_{\mathrm{m}}}{\mathrm{W}_{1}}=\frac{(\mathrm{I}-\mathrm{s})\left(\alpha \cdot \frac{\mathrm{r}_{2}}{\alpha \mathrm{s}+\beta}+\mathrm{r}_{3 \mathrm{~s}}^{*}\right)}{\mathrm{r}_{1} \mathrm{C}_{2 \mathrm{~s}}^{2}+\mathrm{r}_{\mathrm{a}}\left(\frac{\mathrm{Z}_{2 \mathrm{~s}}}{z_{\mathrm{a}}}\right)^{2}+\mathrm{R}_{25}} ;
$$

für die Generatorarbeitsgebiete gilt der reziproke Wert dieses Bruches.

Die aus den Leistungskurven der Abb. 1 I b ermittelten Wirkungsgrade sind in Abb. I I c dargestellt.

Nachstehend sind die in den abgeleiteten Ausdrücken vorkommenden Größen zusammengestellt :

$$
\begin{aligned}
& \mathrm{C}_{1}^{2}=\frac{\left(\mathrm{r}_{1}+\mathrm{r}_{\mathrm{a}}\right)^{2}+\left(\mathrm{x}_{1}+\mathrm{x}_{\mathrm{a}}\right)^{2}}{z_{\mathrm{a}}^{2}}(27 \mathrm{~b}), \\
& \mathrm{r}_{\mathrm{I}}^{*}=\frac{\mathrm{I}}{\mathrm{C}_{1}^{2}} \cdot\left[\mathrm{r}_{1}+\mathrm{r}_{\mathrm{a}}\left(\frac{z_{1}}{z_{\mathrm{a}}}\right)^{2}\right] \\
& \mathrm{x}_{\mathrm{I}}^{*}=\frac{1}{\mathrm{C}_{1}^{2}} \cdot\left[\mathrm{x}_{1}+\mathrm{x}_{\mathrm{a}}\left(\frac{z_{1}}{z_{\mathrm{a}}}\right)^{2}\right]
\end{aligned}
$$

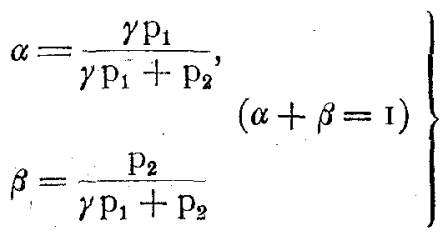

$$
\begin{aligned}
& \left.\begin{array}{l}
\mathrm{R}_{2 \mathrm{~s}}=\frac{\mathrm{r}_{2}}{\alpha \mathrm{s}+\beta}+\mathrm{r}_{3 \mathrm{~s}}^{*}, \\
\mathrm{X}_{2 \mathrm{~s}}=\mathrm{x}_{2}+\mathrm{x}_{3 \mathrm{~s}}^{*}
\end{array}\right\}
\end{aligned}
$$




$$
\begin{aligned}
& \mathrm{r}_{3 \mathrm{~s}}^{*}=\frac{\mathrm{I}}{\mathrm{D}_{3 \mathrm{~s}}^{2}} \cdot \frac{\mathrm{r}_{3 \mathrm{t}}}{\mathrm{s}} \\
& \mathrm{x}_{3 \mathrm{~s}}^{*}=\frac{\mathrm{I}}{\mathrm{D}_{3 \mathrm{~s}}^{2}} \cdot\left[\mathrm{x}_{\mathrm{b}}\left(\frac{\mathrm{z}_{3 \mathrm{~s}}}{\mathrm{x}_{\mathrm{b}}}\right)^{2}+\mathrm{x}_{3}\right] ; \mathrm{z}_{3 \mathrm{~s}}^{2}=\left(\frac{\mathrm{r}_{3 \mathrm{t}}}{\mathrm{s}}\right)^{2}+\mathrm{x}_{3}^{2} \\
& \mathrm{D}_{3 \mathrm{~s}}^{2}=\frac{\left(\frac{\mathrm{r}_{3 \mathrm{t}}}{\mathrm{s}}\right)^{2}+\left(\mathrm{x}_{\mathrm{b}}+\mathrm{x}_{3}\right)^{2}}{\mathrm{x}_{\mathrm{b}}^{2}} \cdot(89) \\
& \mathrm{Z}_{2 \mathrm{~s}}^{2}=\mathrm{R}_{2 \mathrm{~s}}^{2}+\mathrm{X}_{2 \mathrm{~s}}^{2} \\
& \mathrm{C}_{2 \mathrm{~s}}^{2}=\frac{\left(\mathrm{r}_{\mathrm{a}}+\mathrm{R}_{2 \mathrm{~s}}\right)^{2}+\left(\mathrm{x}_{\mathrm{a}}+\mathrm{X}_{2 \mathrm{~s}}\right)^{2}}{\mathrm{z}_{\mathrm{a}}^{2}}
\end{aligned}
$$

Wenn wir in II Ib) $s=$ I setzen, erhalten wir das gesamte Anlaufdrehmoment in synchronen Watt bei kurzgeschlossenem Anlasser

$$
\mathrm{W}_{\mathrm{St}}=\frac{\mathrm{P}_{1}^{2}}{\mathrm{C}_{1}^{2}} \cdot \frac{\alpha \mathrm{r}_{2}+\mathrm{r}_{3 \mathrm{St}}^{*}}{\left(\mathrm{r}_{\mathrm{I}}^{*}+\mathrm{R}_{2 \mathrm{St}}\right)^{2}+\left(\mathrm{X}_{\mathrm{I}}^{*}+\mathrm{X}_{2 \mathrm{St}}\right)^{2}}
$$

welches durch Einschaltung von Widerständen in den Sekundärkreis der hinteren Maschine (Tertiärkreis der Kaskadenschaltung) verändert werden kann. Dabei ist

$$
\begin{aligned}
& \mathrm{R}_{2} \mathrm{St}=\mathrm{r}_{2}+\mathrm{r}_{3}^{*} \mathrm{St}=\mathrm{r}_{2}+\frac{\mathrm{x}_{\mathrm{b}}^{2} \mathrm{r}_{3 \mathrm{t}}}{\mathrm{r}_{3 \mathrm{t}}^{2}+\left(\mathrm{x}_{\mathrm{b}}+\mathrm{x}_{3}\right)^{2}}, \\
& \mathrm{X}_{2 \mathrm{St}}=\mathrm{x}_{2}+\mathrm{x}_{3 \mathrm{St}}^{*}=\mathrm{x}_{2}+\frac{\mathrm{x}_{\mathrm{b}}\left(\mathrm{r}_{3 \mathrm{t}}^{\mathrm{a}}+\mathrm{x}_{3}^{2}\right)+\mathrm{x}_{\mathrm{b}}^{\mathrm{a}} \mathrm{x}_{3}}{\mathrm{r}_{3 \mathrm{t}}^{2}+\left(\mathrm{x}_{\mathrm{b}}+\mathrm{x}_{3}\right)^{2}} .
\end{aligned}
$$

Schluß3bemerkung. Das in den vorstehenden Abschnitten entwickelte Verfahren ermöglicht auch eine verhältnismäßig einfache Untersuchung der Anlaufverhältnisse deŕ Kaskadenschaltung (Abhängigkeit des Anlaufdrehmoments von den in den Sekundärkreis der hinteren Maschine eingeschalteten Widerständen), sowie des Einflusses der dauernden Einschaltung von Widerständen in den Sekundärkreis der hinteren Maschine. Diese Fragen sollen an einer anderen Stelle behandelt werden.

Die Konstruktion des Leitwert-(Strom-)Diagramms der Kaskadenschaltung wird wesentlich vereinfacht, wenn die früher berücksichtigten Eisenverluste und die Kupferverluste in der Primärwicklung der vorderen Maschine vernachlässigt werden. Wir erhalten damit ein Diagramm, welches in seinen Voraussetzungen dem bekannten Heylanddiagramm der einfachen Induktionsmaschine entspricht. 